\title{
Frame-Based Filter-Function Formalism for Quantum Characterization and Control
}

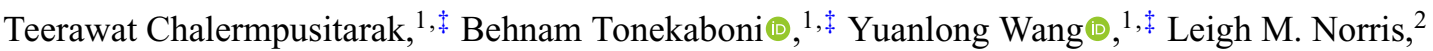 \\ Lorenza Viola, ${ }^{2, \dagger}$ and Gerardo A. Paz-Silva ${ }^{1, *}$ \\ ${ }^{1}$ Centre for Quantum Computation and Communication Technology (Australian Research Council), \\ Centre for Quantum Dynamics, Griffith University, Brisbane, Queensland 4111, Australia \\ ${ }^{2}$ Department of Physics and Astronomy, Dartmouth College, 6127 Wilder Laboratory, Hanover, New Hampshire \\ 03755, USA
}

(Received 20 September 2020; revised 24 May 2021; accepted 28 June 2021; published 27 July 2021)

We introduce a theoretical framework for resource-efficient characterization and control of nonMarkovian open quantum systems, which naturally allows for the integration of given, experimentally motivated, control capabilities and constraints. This is achieved by developing a transfer filter-function formalism based on the general notion of a frame and by appropriately tying the choice of frame to the available control. While recovering the standard frequency-based filter-function formalism as a special instance, this control-adapted generalization affords intrinsic flexibility and, crucially, it permits an efficient representation of the relevant control matrix elements and dynamical integrals if an appropriate finite-size frame condition is obeyed. Our frame-based formulation overcomes important limitations of existing approaches. In particular, we show how to implement quantum noise spectroscopy in the presence of nonstationary noise sources, and how to effectively achieve control-driven model reduction for noise-optimized prediction and quantum gate design.

DOI: 10.1103/PRXQuantum.2.030315

\section{INTRODUCTION}

Accurate characterization and control $(\mathrm{C} \& \mathrm{C})$ of open quantum systems coupled to realistic - temporally correlated ("non-Markovian") — noise environments are vital for exploiting the full potential of quantum technologies. Open-loop control-engineering methods, based on tailored time-dependent modification of the open-system dynamics, offer a versatile and experimentally accessible approach to tackle this challenge. While techniques employing dynamical decoupling or dynamically corrected gates [1-3] are beneficial under minimal knowledge about the noise-inducing degrees of freedom, extra knowledge is instrumental for optimizing their performance and efficiency in specific noise environments $[4,5]$. Numerical quantum optimal control algorithms [6-8]

\footnotetext{
*g.pazsilva@griffith.edu.au

†lorenza.viola@dartmouth.edu

\$These authors contributed equally to this work.
}

Published by the American Physical Society under the terms of the Creative Commons Attribution 4.0 International license. Further distribution of this work must maintain attribution to the author(s) and the published article's title, journal citation, and DOI. represent an extreme example: although they can in principle deliver exceptionally high fidelities, their viability in non-Markovian settings requires detailed knowledge of the time-domain noise correlation functions (or the corresponding frequency-domain spectra) [9]. In addition to permitting noise-optimized quantum storage and gate design, accurate noise characterization is key to counter the effect of non-Markovian noise in quantum sensing and metrology applications [10,11].

This has motivated the development of "quantum noise spectroscopy" (QNS) protocols, in which noise spectral information is inferred from the system-only reduced dynamics, under the effects of the noise and user-defined control. Despite considerable progress [12-26], existing QNS protocols suffer from several disadvantages. First, they are not applicable to important types of noise that occur in practice - notably, nonstationary noise, for which a frequency-domain description need not be viable [27, 28]. Second, they do not easily lend themselves to the identification of simplified noise models that, while providing all the required detail for optimal control to be feasible, may permit $\mathrm{C} \& \mathrm{C}$ procedures to be extensible to increasingly larger qubit networks. Finally, to the best of our knowledge, no formal analysis of the "universality" of QNS-inferred information for control purposes has been attempted, aimed at clarifying the extent to which 
such information may suffice to faithfully predict the dynamics of the system under an arbitrary control modulation, beyond what is used in the QNS protocol itself.

In this paper, we overcome the above limitations by demonstrating how a "model-reduced" representation of the noisy dynamics of interest may be tied to the available, finite control resources. We achieve this by integrating the language of frames [29-33] - already a mainstay in signal processing - with the theory of open quantum systems. This leads to a novel transfer filter-function (FF) formalism [34-36], in which control capabilities and constraints (dubbed $\mathscr{C}$ henceforth) play the defining role. Crucially, our framework allows the identification of the noise components relative to $\mathscr{C}$ that are, at once, accessible to estimation by a QNS protocol and sufficient to optimally predict the behavior of the system. Moreover, it provides a rigorous way to determine (and quantify) if the information inferred via QNS is useful to predict the behavior of the system under a given control sequence.

The content is organized as follows. In Sec. II, we introduce the relevant setting for controlled non-Markovian quantum dynamics, with special emphasis on highlighting the structure of the perturbative overlap integrals that enter any $\mathrm{C} \& \mathrm{C}$ protocol. Section III provides the conceptual foundation of our approach: after providing the essential mathematical background on frames, we show how the relationship between a frame $\mathscr{F}$ and its canonical dual $\tilde{\mathscr{F}}$ naturally suggests two complementary ways for separating the dynamical overlap integrals into control-dependent and noise-dependent contributions, resulting in what we call the standard picture versus the control-adapted picture, relative to a chosen $(\mathscr{F}, \tilde{\mathscr{F}})$ pair. In particular, we recover the usual frequency-domain FF formalism as a special instance associated with the use of a Fourier frame in Sec. III B, whereas in Sec. III C we make precise the sense in which control-driven model reduction may be achieved, provided that a suitable finite-size frame condition is obeyed. In essence, the latter ensures that arbitrary control sequences built out of $\mathscr{C}$ may be well approximated by finite expansions over elements of $\mathscr{F}$.

We then proceed to showcase the added generality and flexibility of our approach by focusing, in Sec. IV, on multiqubit dynamics under simultaneous additive decoherence and multiplicative control noise. After providing a general frame construction for this setting, we specialize to the simplest paradigmatic case of single-qubit dynamics. In particular, in Sec. IV B we demonstrate how controladapted QNS techniques that are designed to work directly in the time domain provide new capabilities over existing protocols, by allowing the characterization of nonstationary noise environments of both classical and genuinely quantum nature. A complete frame-based C\&C protocol is exemplified in Sec. IV C, where the information about the noise correlations acquired through a first stage of control-adapted QNS is subsequently incorporated in the optimal-control search for various target unitary gates. By comparing to the optimal-control solutions obtained under access to the full dynamics, we establish that our model-reduced representation incurs no significant loss of predictive power, insofar as arbitrary controlled evolutions built out of $\mathscr{C}$ are considered. Finally, we present in Sec. IV D some important remarks on the extent to which QNS-inferred information may be universal for prediction and control, before concluding. Further technical detail is included in five separate appendices, in order to make the presentation as self-contained as possible.

\section{CONTROLLED OPEN QUANTUM DYNAMICS}

We consider a controlled $d$-dimensional open quantum system $S$ evolving in the presence of an inaccessible environment (bath) $B$. In the interaction picture associated with the free bath evolution, the joint system-bath dynamics is governed by a Hamiltonian of the form $H(t)=H_{S}+$ $H_{S B}(t)+H_{\mathrm{ctrl}}(t)$, where the Hamiltonian $H_{S}$ determines the free evolution of $S, H_{S B}(t)$ couples $S$ and $B$, and $H_{\text {ctrl }}(t)$ describes open-loop control modulation acting on $S$ only. Let $\left\{\Lambda_{0} \equiv I_{S}, \Lambda_{u}\right\}$ denote a generalized (Hermitian) Pauli basis for the operator space on $S$, with $\operatorname{Tr}\left(\Lambda_{u} \Lambda_{v}\right)=\delta_{u v}$. We consider a broad class of Hamiltonians that simultaneously account for additive (control-independent) and multiplicative (control-dependent) noise, according to

$$
H(t)=\sum_{u \neq 0} \Lambda_{u} \otimes B_{u}^{(a)}(t)+\sum_{v \neq 0} h_{v}(t)\left[1+\beta_{v}^{(m)}(t)\right] \Lambda_{v} .
$$

Here, the $B_{u}^{(a)}(t) \equiv \tilde{B}_{u}^{(a)}(t)+\beta_{u}^{(a)}(t) I_{B}$ describe the alwayson additive $(a)$ noise from quantum and classical sources, respectively, with $\tilde{B}_{u}^{(a)}(t)$ being bath operators (not necessarily traceless in order to account for evolution due to $H_{S}$ ) and $\beta_{u}^{(a)}(t)$ stochastic processes. Control is introduced via user-defined control profiles $\left\{h_{v}(t)\right\}$ that, subject to systemdependent constraints (e.g., maximum amplitude, finite time-bandwidth product), determine the control capabilities $\mathscr{C}$ in the error-free scenario. More precisely, we define the control capabilities $\mathscr{C}$ as the set of control Hamiltonians $\left\{h_{v}(t) \Lambda_{v}\right\}_{v \neq 0}$ that can be implemented in a given experiment. There are two aspects to this: the allowed control "directions" $\Lambda_{v}$ and the "control profiles" $h_{v}(t)$, usually parametrically defined by a pulse shape and a range for the specifying parameters, e.g., $h_{v}(t) \sim \theta e^{-t^{2} / 2 \sigma^{2}}$ for $\theta \in\left[\theta_{\min }, \theta_{\max }\right]$ and $\sigma \in\left[\sigma_{\min }, \sigma_{\max }\right]$. Furthermore, multiplicative $(m)$ control noise, which often arises in realistic settings [16,20,37], is captured by the stochastic processes $\beta_{v}^{(m)}(t)$

Our main objects of interest are the time-dependent expectation values of system observables $O=O^{\dagger}$, given 
by

$$
E[O(T)]_{\rho_{S B}}=\left\langle\operatorname{Tr}_{S, B}\left[U(T) \rho_{S B} U^{\dagger}(T)\left(O \otimes I_{B}\right)\right]\right\rangle_{c},
$$

where $\rho_{S B}$ is the initial state of the joint system and bath. To obtain useful expressions for them we proceed as follows. The unitary propagator for the evolution generated by $H(t)$ is given (in units $\hbar=1$ ) by the timeordered exponential $U(t)=\mathcal{T}_{+} e^{-i \int_{0}^{t} d s H(s)}$, and $\langle\cdot\rangle_{c}$ denotes the average over realizations of the stochastic processes. We may write $H(t) \equiv H_{0}(t)+H_{e}(t)$ with $H_{0}(t)$ representing the intended, error-free controlled dynamics and the error component $H_{e}(t)$ accounting for unwanted evolution due to environmental and control noise, as well as possibly $H_{S}$ [e.g., $H_{0}(t)=H_{\text {ctrl }}(t)$ if $H_{S}=0$ and no control noise is present, $\left.\beta_{v}^{(m)}(t)=0\right]$. The evolution due to $H_{e}(t)$ can then be isolated by moving to the interaction (toggling) frame associated with the error-free component $H_{0}(t)$. That is, we let $U(t)=U_{0}(t) \widetilde{U}(t)$, where

$$
U_{0}(t)=\mathcal{T}_{+} e^{-i \int_{0}^{t} d s H_{0}(s)}, \quad \widetilde{U}(t)=\mathcal{T}_{+} e^{-i \int_{0}^{t} d s \widetilde{H}(s)},
$$

and $\widetilde{H}(t) \equiv U_{0}^{\dagger}(t) H_{e}(t) U_{0}(t)$ is the toggling-frame Hamiltonian. By construction, such a Hamiltonian vanishes in the absence of noise, and can be compactly written as

$$
\widetilde{H}(t) \equiv \sum_{\alpha=a, m} \sum_{u, v} y_{u, v}^{(\alpha)}(t) \Lambda_{v} \otimes B_{u}^{(\alpha)}(t),
$$

where we have defined $B_{u}^{(m)}(t) \equiv \beta_{u}^{(m)}(t) I_{B}$ and the elements $y_{u, v}^{(\alpha)}(t)$ of the control matrix $\mathscr{Y}^{(\alpha)}(t)$ capture the effect of the control modulation on the noise terms in $H_{e}(t)$. Explicitly, we have

$$
\begin{aligned}
& y_{u, v}^{(a)}(t)=\operatorname{Tr}_{S}\left[U_{0}^{\dagger}(t) \Lambda_{u} U_{0}(t) \Lambda_{v}\right] / d, \\
& y_{u, v}^{(m)}(t)=h_{u}(t) y_{u, v}^{(a)}(t) .
\end{aligned}
$$

Finally, assuming that $\rho_{S B}=\rho_{S} \otimes \rho_{B}$ (see Ref. [38] for a more general treatment) and that $O$ is invertible, with $\widetilde{O}(T) \equiv U_{0}^{\dagger}(T) O U_{0}(T)$, we write the desired timedependent expectation value, Eq. (2), as

$$
E[O(T)]_{\rho_{S} \otimes \rho_{B}}=\operatorname{Tr}_{S}\left[V_{O}(T) \rho_{S} \widetilde{O}(T)\right],
$$

where the system operator

$$
V_{O}(T) \equiv\left\langle\operatorname{Tr}_{B}\left[\widetilde{O}^{-1}(T) \widetilde{U}^{\dagger}(T) \widetilde{O}(T) \widetilde{U}(T) \rho_{B}\right]\right\rangle_{c}
$$

contains all the unwanted effects due to $H_{e}(t)$ up to time $T$; that is, $V_{O}(T)=I_{S}$ represents ideal, noise-free dynamics.

The operator $V_{O}(T)$ may be computed perturbatively, for instance through a Dyson or cumulant expansion [24,39]. Regardless of what technique is chosen it can be shown that, due to time ordering, the dynamics depends only on certain linear combinations, say $\mathcal{L}_{\vec{v}}(\vec{t})$, of the multitime noise correlation functions $\left\{\left\langle B_{v_{1}}^{\left(\alpha_{1}\right)}\left(t_{\mu(1)}\right) \cdots B_{v_{k}}^{\left(\alpha_{k}\right)}\left(t_{\mu(k)}\right)\right\rangle\right\}$ with respect to the combined quantum and classical averages, $\langle\cdot\rangle \equiv\left\langle\operatorname{Tr}_{B}\left[\cdot \rho_{B}\right]\right\rangle_{c}$, with $\alpha_{j} \in\{a, m\}$ and $\mu$ being an arbitrary permutation of $k$ elements, $k \in \mathbb{N}$. While the specifics of the linear combinations that enter the expectations in Eq. (4) depend on both $O$ and the details of the system and control setting, the possible contributions are determined by overlap integrals of the form (see Appendix A)

$$
\mathcal{I}_{\vec{\alpha} ; \vec{u}, \vec{v}}^{(k)}(T)=\int_{0}^{T} d_{>} \vec{t}_{[k]}\left[\prod_{j=1}^{k} y_{u_{j}, v_{j}}^{\left(\alpha_{j}\right)}\left(t_{j}\right)\right] \mathcal{L}_{\vec{\alpha} ; \vec{v}}(\vec{t})
$$

with $\int_{0}^{T} d_{>} \vec{t}_{[k]} \equiv \int_{0}^{T} d t_{1} \int_{0}^{t_{1}} d t_{2} \cdots \int_{0}^{t_{k-1}} d t_{k}$.

These integrals are key to understanding the effect of the noise on the system. The conventional approach to their analysis involves rewriting them as multidimensional overlap integrals in frequency space via an appropriate Fourier transform. This leads to the standard FF formalism [34-36], where frequency-domain FFs and, for general non-Gaussian noise, higher-order (poly)spectra capture the effect of the control and noise, respectively. On the one hand, one expects that, by (experimentally) obtaining the value of any such integral for various FFs, it may be in principle possible to deconvolve it, and thus infer relevant information about the noise correlation functions (or their Fourier transform). This is indeed how QNS protocols work. On the other hand, one also sees that mitigating the effect of the noise is akin to minimizing the value of such integrals, which is the working principle behind existing decoherence suppression and optimal control protocols. The frequency- (or time-)domain representations, however, are agnostic to the control capabilities or constraints $\mathscr{C}$, which are unavoidable in any realistic setting, since the $\left\{h_{v}(t)\right\}$ cannot be arbitrarily chosen. Indeed, these constraints translate into limitations to the information that can be inferred about the noise (e.g., in comb-based QNS protocols the frequency-domain spectra are sampled via a discrete grid), or superfluous information used for synthesizing optimal control (e.g., rather than full knowledge of the noise correlation functions, only knowledge of the overlap between noise and the admissible controls should be necessary). This motivates the search for a space - or a mathematical language - in which the relevant overlap integrals can be more efficiently represented by incorporating information about $\mathscr{C}$ from the outset.

\section{FRAME-BASED FILTER FUNCTIONS AND CONTROL-DRIVEN MODEL REDUCTION}

In order to meet the challenge identified in the previous section, instead of moving to the frequency domain we use the more general language of frames [29-33]. 
Frames have a long tradition in signal processing, thanks to the flexibility they afford as compared to bases (e.g., in generalizing time-to-frequency-domain transforms) as well as to various properties particularly advantageous to signal reconstruction (e.g., robustness to noise [40]). Moreover, they are already successfully exploited in different quantum applications [38,41,42]. Leveraging the frame language in our context will first and foremost afford extra flexibility, as a frame can be chosen in a way that matches - in a sense that will be made precise later - the available control. In turn, aided by a change in point of view, this will allow us to efficiently represent each of the overlap $\mathcal{I}^{(k)}$ integrals as a sum over a finite domain, thereby achieving control-driven model reduction.

\section{A. Basics on frames}

The mathematical theory of frames is quite sophisticated and an exhaustive review of the topic is beyond our scope. While we refer the interested reader to the extensive literature for a more complete and rigorous account $[30,31,33]$, we summarize here the basic definitions needed for the exposition of our result. We further discuss in Appendix B illustrative examples directly relevant to the control scenarios we analyze.

Let $\mathcal{H}$ be a complex (finite-dimensional or separable) Hilbert space, consisting of functions $f(t), t \in[0, T]$, with inner product and norm respectively given by

$$
(f, g) \equiv \frac{1}{T} \int_{0}^{T} d t f(t) g^{*}(t), \quad\|f\|^{2} \equiv(f, f) .
$$

A discrete frame for $\mathcal{H}$ is an (at most) countable sequence $\mathscr{F} \equiv\left\{\phi_{n}\right\}_{n}$, with $\phi_{n} \in \mathcal{H}$ and $n \in \mathbb{Z}$, satisfying the frame condition, that is,

$$
A\|f\|^{2} \leq \sum_{n}\left|\left(f, \phi_{n}\right)\right|^{2} \leq B\|f\|^{2} \quad \text { for all } f \in \mathcal{H}
$$

with $0<A \leq B<\infty$ being the lower and upper frame bounds, respectively. Of particular interest are tight frames, for which $A=B$, and Parseval frames, for which, in addition, $A=B=1$. Notably, an orthonormal basis in $\mathcal{H}$ is a Parseval frame, and indeed one sees that in this case the frame condition is equivalent to Parseval's identity.

Frames may be seen as redundant (linearly dependent) spanning sets for $\mathcal{H}$. More precisely, any $f \in \mathcal{H}$ can be expanded as $f(t)=\sum_{n} c_{n} \phi_{n}(t), t \in[0, T]$, where the coefficients are given by the reconstruction formula

$$
f(t)=\sum_{n}\left(f, \tilde{\phi}_{n}\right) \phi_{n}(t)=\sum_{n}\left(f, \phi_{n}\right) \tilde{\phi}_{n}(t)
$$

and the $\left\{\tilde{\phi}_{n}\right\}_{n}$ are elements of a frame dual to $\mathscr{F}$. While a frame $\mathscr{F}$ may in general admit infinitely many dual frames, there exists a canonical dual frame $\tilde{\mathscr{F}}$ that is special, in the sense that it minimizes the norm of the expansion

$$
\sum_{n}\left|\left(f, \tilde{\phi}_{n}\right)\right|^{2} \leq \sum_{n}\left|c_{n}\right|^{2}
$$

for any sequence $\left\{c_{n}\right\}_{n} \in \ell_{2}(\mathbb{Z})$ that satisfies $f=\sum_{n} c_{n} \phi_{n}$, with equality holding if and only if $c_{n}=\left(f, \tilde{\phi}_{n}\right)$ for all $n$. Such a dual frame is determined by $\tilde{\mathscr{F}}=\left\{\tilde{\phi}_{n}\right\}=\left\{\mathbf{S}^{-1} \phi_{n}\right\}$, in terms of the positive frame operator $\mathbf{S}: \mathcal{H} \rightarrow \mathcal{H}$, given by

$$
\mathbf{S} f \equiv \sum_{n}\left(f, \phi_{n}\right) \phi_{n}
$$

Note that $\mathbf{S}$ is a multiple of the identity if the frame is tight and it coincides with the identity for a basis, that is, an orthonormal basis is self-dual. Of special relevance to this work will be finite frames, $\left\{\phi_{n}\right\}_{n}, n=1, \ldots, N_{\#}<\infty$.

More generally, continuous frames for which the labeling index $n \mapsto x \in X$ changes continuously can also be constructed. Given a measure space $(X, \mathscr{W}, \mu)$, a family $\mathscr{F} \equiv\left\{\phi_{x}\right\}_{x}$ is a continuous frame if (i) for all $f \in \mathcal{H}$, $\left(f, \phi_{x}\right)$ is $\mathscr{W}$ measurable in $X$ and (ii) there exist constants $0<A \leq B<\infty$ such that

$$
A\|f\|^{2} \leq \int_{X}\left|\left(f, \phi_{x}\right)\right|^{2} d \mu(x) \leq B\|f\|^{2} \quad \text { for all } f \in \mathcal{H} .
$$

From the above, it follows that $\overline{\operatorname{span}}\left\{\left(\phi_{x}\right)\right\}_{x \in X}=\mathcal{H}$, and an appropriate notion of dual frame and the reconstruction formula can be introduced by essentially replacing sums - as in Eq. (8) - with integrals of the form $\int_{X} d \mu(x)$. In this way, the special case of a discrete frame is recovered when $\mu$ is a counting measure and $X=\mathbb{Z}$ (or $\mathbb{N}$ ). Prominent examples of continuous frame expansions are expansions into canonical and generalized coherent states and the continuous short-time Fourier (or Gábor) transform [33], whereas Fourier series are a special case of expansions into discrete exponential (or Fourier) frames $[30,31]$.

\section{B. Standard-picture versus control-adapted filters and spectra}

To apply the frame formalism to our problem, we start by noting that there are two ways of representing any of the time-ordered integrals of Eq. (6). On the one hand, we can write the noise correlation function in the chosen frame and rewrite the remaining factors as a FF associated with that frame. We dub this the "standard picture" (SP). On the other hand, we may expand the control matrix elements in the frame and have the remaining factors be the equivalent of a noise spectra in the frame language - resulting in 
what we dub the "control-adapted" (CA) picture. Mathematically, these two approaches respectively lead to

$$
\begin{aligned}
\mathcal{I}_{\vec{\alpha} ; \vec{u}, \vec{v}}^{(k)}(T) & =\sum_{\vec{n}} F_{\vec{\alpha} ; \vec{u}, \vec{v}}^{(k)}(\vec{n}, T) S_{\vec{\alpha} ; \vec{v}}^{(k)}(\vec{n}) \\
& =\sum_{\vec{n}}\left[\prod_{j=1}^{k} F_{\alpha_{j} ; u_{j}, v_{j}}^{(1)}\left(n_{j}, T\right)\right] \bar{S}_{\vec{\alpha} ; \vec{v}}^{(k)}(\vec{n}) .
\end{aligned}
$$

Equation (10a) represents the direct generalization to the (discrete) frame language of the standard frequencydomain representation: the $k$ th-order frame-based FFs and frame-based power spectra are respectively given by

$$
\begin{gathered}
F_{\vec{\alpha} ; \vec{u}, \vec{v}}^{(k)}(\vec{n}, T) \equiv \int d_{>} \vec{t}_{[k]} \prod_{j} y_{u_{j}, v_{j}}^{\left(\alpha_{j}\right)}\left(t_{j}\right) \tilde{\phi}_{n_{j}}^{\left(\alpha_{j}\right)}\left(t_{j}\right)^{*}, \\
S_{\vec{\alpha} ; \vec{v}}^{(k)}(\vec{n})=\int \overrightarrow{d t}_{[k]} \mathcal{L}_{\vec{\alpha} ; \vec{v}}(\vec{t}) \prod_{i=1}^{k} \phi_{n_{i}}^{\left(\alpha_{i}\right)}\left(t_{i}\right),
\end{gathered}
$$

where we allow for different frames $\mathscr{F}^{(\alpha)}$ for $\alpha \in\{a, m\}$. Indeed, as shown explicitly in Appendix B 1, the standard frequency-domain FF formalism [34,36] is recovered when one uses a Fourier frame [43]. In contrast, Eq. (10b) does not only represent a generalization to the frame language, but, importantly for our purposes, it also provides a change in viewpoint - a dual representation in which

$$
\begin{aligned}
F_{\alpha_{j} ; u_{j}, v_{j}}^{(1)}\left(n_{j}, T\right) & \equiv\left(y_{u_{j}, v_{j}}^{\left(\alpha_{j}\right)}, \tilde{\phi}_{n_{j}}^{\left(\alpha_{j}\right)}\right) \\
& =\int_{0}^{T} d t y_{u_{j}, v_{j}}^{\left(\alpha_{j}\right)}(t) \tilde{\phi}_{n_{j}}^{\left(\alpha_{j}\right)}(t)^{*}, \\
\bar{S}_{\vec{\alpha} ; \vec{v}}^{(k)}(\vec{n}) & =\int d_{>} \vec{t}_{[k]} \mathcal{L}_{\vec{\alpha} ; \vec{v}}(\vec{t}) \prod_{i=1}^{k} \phi_{n_{i}}^{\left(\alpha_{i}\right)}\left(t_{i}\right),
\end{aligned}
$$

are the frame-based fundamental FFs and the frame-based $C A$ spectra, respectively. Note that, in the CA representation, the time ordering is moved from the FFs to the CA spectra, with a twofold implication. On the one hand, as in the SP setting, the CA spectra encode all the information about noise correlations that influence the system dynamics, as we explicitly demonstrate in Sec. IV. On the other hand, the fact that the CA FFs are all one-dimensional integrals without any time ordering makes their use more advantageous to both theoretical analysis and numerical implementation of C\&C.

We also note that there is a degree of arbitrariness in the above definitions since, given a frame $\mathscr{F}=\left\{\phi_{n}\right\}$ and its dual $\tilde{\mathscr{F}}=\left\{\tilde{\phi}_{n}\right\}$, their corresponding complex conjugates (say $\mathscr{F}^{*}$ and $\tilde{\mathscr{F}}^{*}$ ) are also frames. In order to maintain a certain symmetry in our expressions, in this paper we choose to expand in $\tilde{\mathscr{F}}^{*}$ and $\mathscr{F}$ in order to respectively generate the SP and CA formalisms. For completeness, we summarize the resulting expressions in Table I.

\section{Frame-based control-driven model reduction}

The next key step in our approach is to observe that, as far as the effect of the noise on the system is concerned [captured by the operator $V_{O}$ in Eq. (5)], what matters are not the control inputs $\left\{h_{u}(t)\right\}$ themselves, but rather their control matrix elements $\left\{y_{u, v}^{(\alpha)}(t)\right\}$, which are related to the $\left\{h_{u}(t)\right\}$ via conjugation under the known map $U_{0}(t)$. Moreover, any constraints on $h_{u}(t)$ (say, limited bandwidth, a minimum time between two consecutive pulses, or a finite time resolution) necessarily translate into limitations on the possible form that the $y_{u, v}^{(\alpha)}(t)$ can take.

These observations, the flexibility of frames, and the CA representation of $\mathcal{I}_{u, v}^{(k)}$ come together as follows: (i) for given control capabilities $\mathscr{C}$, the possible $\left\{y_{u, v}^{(\alpha)}(t)\right\}$ are known; (ii) it is in principle possible to tailor the choice of frame $\mathscr{F}^{(\alpha)}$ so that it "efficiently" represents such $\left\{y_{u, v}^{(\alpha)}(t)\right\}$; (iii) this, in turn, leads to an "efficient" representation of the integrals in $\mathcal{I}_{u, v}^{(k)}$. More formally, we introduce the following condition.

Definition (Finite-size frame (FSF) condition). Let $\mathscr{C}$ specify fixed control capabilities, which determine a (possibly infinite) set of control matrix elements, $y_{u, v}^{(\alpha)}(t) \in$ $L^{2}([0, T]), \alpha \in\{a, m\}$. We say that the FSF condition holds if one can build finite-size frames $\mathscr{F}_{\#}^{(\alpha)} \equiv\left\{\phi_{n}^{(\alpha)}\right\}$, $n=1, \ldots, N_{\#}^{(\alpha)}$, and dual frame $\tilde{\mathscr{F}}_{\#}^{(\alpha)}$, such that, for all $y_{u, v}^{(\alpha)}(t)$ allowed by $\mathscr{C}$,

$$
y_{u, v}^{(\alpha)}(t)=\sum_{n=1}^{N_{\sharp}^{(\alpha)}} F_{\alpha ; u, v}^{(1)}(n, t) \phi_{n}^{(\alpha)}(t) .
$$

We say that the FSF condition is satisfied to tolerance $\varepsilon \geq 0$ over $[0, T]$ if the above equality can be approximately obeyed with error no larger than $\varepsilon$ (in the $L^{2}$ norm).

If the FSF condition holds, the $\left\{y_{u, v}^{(\alpha)}(t)\right\}$ are represented efficiently in the sense that they are well approximated by a finite expansion over the elements of $\tilde{\mathscr{F}}_{\#}^{(\alpha)}$. It then follows that

$$
\mathcal{I}_{\vec{\alpha} ; \vec{u}, \vec{v}}^{(k)}(T) \simeq \sum_{n_{i}=1}^{N_{\#}^{\left(\alpha_{j}\right)}}\left(\prod_{j} F_{\alpha_{j} ; u_{j}, v_{j}}^{(1)}\left(n_{j}\right)\right) \bar{S}_{\vec{\alpha} ; \vec{v}}^{(k)}(\vec{n}),
$$

that is, each integral can be efficiently represented by a finite sum up to an error that scales as $\mathcal{O}(\varepsilon k)$. A key consequence of the above is that it allows us to identify the components of the noise that are relevant to the dynamics allowed by $\mathscr{C}$ : namely, the finite set of CA spectra,

$$
\overline{\mathscr{S}}_{\mathscr{C}}=\left\{\bar{S}_{\vec{\alpha} ; \vec{v}}^{(k)}(\vec{n})\right\}, \quad n_{j} \in\left[1, N_{\#}^{\left(\alpha_{j}\right)}\right]
$$

(or specific combinations thereof), that contribute to the expectations of observables as in Eq. (4). Thus, $\left.\overline{\mathscr{S}}\right|_{\mathscr{C}}$ 
TABLE I. Summary of the defining relationships for standard (i) versus control-adapted (ii) pictures. The corresponding frequencydomain SP and CA representations are also included in (iii) and (iv) for comparison.

\begin{tabular}{|c|c|}
\hline (i) $\mathrm{SP}$ & (ii) $\mathrm{CA}$ \\
\hline $\mathcal{L}_{\vec{\alpha}, \vec{v}}(\vec{t})=\sum_{\vec{n}}\left(\mathcal{L}_{\vec{\alpha}, \vec{v}}(\vec{s}), \prod_{i} \phi_{n_{i}}^{\left(\alpha_{j}\right)}\left(s_{i}\right)^{*}\right) \prod_{j} \tilde{\phi}_{n_{j}}^{\left(\alpha_{j}\right)}\left(t_{j}\right)^{*}$ & $\prod_{j} y_{u_{j}, v_{j}}^{\left(\alpha_{j}\right)}\left(t_{j}\right)=\prod_{j} \sum_{\vec{n}}\left(y_{u_{j}, v_{j}}^{\left(\alpha_{j}\right)}\left(s_{j}\right), \tilde{\phi}_{n_{j^{\prime}}}^{\left(\alpha_{j}\right)}\left(s_{j}\right)\right) \phi_{n_{j^{\prime}}}^{\left(\alpha_{j}\right)}\left(t_{j}\right)$ \\
\hline$\equiv \sum_{\vec{n}} S_{\vec{\alpha} ; \vec{v}}^{(k)}(\vec{n}) \prod_{j} \tilde{\phi}_{n_{j}}^{\left(\alpha_{j}\right)}\left(t_{j}\right)^{*}$ & $\equiv \prod_{j} \sum_{\vec{n}} F_{\alpha_{j} ; u_{j}, v_{j}}^{(1)}\left(n_{j^{\prime}}\right) \phi_{n_{j^{\prime}}}^{\left(\alpha_{j}\right)}\left(t_{j}\right)$ \\
\hline$F_{\vec{\alpha}: \vec{u}, \vec{v}}^{(k)}(\vec{n})=\int d_{>} \vec{t}_{[k]} \prod_{j} y_{u_{j}, v_{j}}^{\left(\alpha_{j}\right)}\left(t_{j}\right) \tilde{\phi}_{n_{j}}^{\left(\alpha_{j}\right)}\left(t_{j}\right)^{*}$ & $F_{\alpha_{j} ; u_{j}, v_{j}}^{(1)}\left(n_{j}\right)=\int d t y_{u_{j}, v_{j}}^{\left(\alpha_{j}\right)}(t) \tilde{\phi}_{n_{j}}^{\left(\alpha_{j}\right)}(t)^{*}$ \\
\hline$S_{\vec{\alpha} ; \vec{v}}^{(k)}(\vec{n})=\int \overrightarrow{d t}_{[k]} \mathcal{L}_{\vec{\alpha} ; \vec{v}}(\vec{t}) \prod \phi_{n_{i}}^{\left(\alpha_{i}\right)}\left(t_{i}\right)$ & $\bar{S}_{\vec{\alpha} ; \vec{v}}^{(k)}(\vec{n})=\int d_{>} \vec{t}_{[k]} \mathcal{L}_{\vec{\alpha}, \vec{v}}(\vec{t}) \prod \phi_{n_{i}}^{\left(\alpha_{i}\right)}\left(t_{i}\right)$ \\
\hline $\mathcal{I}_{\vec{\alpha} ; \vec{u}, \vec{v}}^{(k)}=\sum_{\vec{n}} F_{\vec{\alpha} ; \vec{u}, \vec{v}}^{(k)}(\vec{n}) S_{\vec{\alpha} ; \vec{v}}^{(k)}(\vec{n})$ & $\mathcal{I}_{\vec{\alpha} ; \vec{u}, \vec{v}}^{(k)}=\sum_{\vec{n}} \prod_{j} F_{\alpha_{j} ; u_{j}, v_{j}}^{(1)}\left(n_{j}\right) \bar{S}_{\vec{\alpha} ; \vec{v}}^{(k)}(\vec{n})$ \\
\hline (iii) Frequency domain (SP) & (iv) Frequency domain (CA) \\
\hline$F_{\vec{\alpha} ; \vec{u}, \vec{v}}^{(k)}(\vec{\omega})=\int_{0}^{T} d_{>} \vec{t}_{[k]} \prod_{i=1}^{k} y_{u_{j}, v_{j}}^{\left(\alpha_{j}\right)}\left(t_{j}\right) e^{i \vec{\omega} \cdot \vec{t}}$ & $F_{\alpha_{j} ; u_{j}, v_{j}}^{(1)}(\omega)=\int_{0}^{T} d t y_{u_{j}, v_{j}}^{\left(\alpha_{j}\right)}(t) e^{i \omega t}$ \\
\hline$\left.S_{\vec{\alpha} ; \vec{v}}^{(k)}(\vec{\omega})=\int^{\infty} \overrightarrow{d t}_{[k]} \mathcal{L}_{\vec{\alpha}, \vec{v}} \vec{t}\right) e^{-i \vec{\omega} \cdot \vec{t}}$ & $\left.\bar{S}_{\vec{\alpha} ; \vec{v}}^{(k)}(\vec{\omega})=\int^{\infty} d_{>} \vec{t}_{[k]} \mathcal{L}_{\vec{\alpha}, \vec{v}} \vec{t}\right) e^{-i \vec{\omega} \cdot \vec{t}}$ \\
\hline $\mathcal{I}_{\vec{\alpha} ; \vec{u}, \vec{v}}^{(k)}=\int_{-\infty}^{\infty} d \vec{\omega} F_{\vec{\alpha} ; \vec{u}, \vec{v}}^{(k)}(\vec{\omega}) S_{\vec{\alpha} ; \vec{v}}^{(k)}(\vec{\omega})$ & $\mathcal{I}_{\vec{\alpha} ; \vec{u}, \vec{v}}^{(k)}=\int_{-\infty}^{\infty} d \vec{\omega}\left(\prod_{j} F_{\alpha_{j} ; u_{j}, v_{j}}^{(1)}\left(\omega_{j}\right)\right) \bar{S}_{\vec{\alpha} ; \vec{v}}^{(k)}(\vec{\omega})$ \\
\hline
\end{tabular}

represents both the information that can be extracted from the reduced system dynamics, and what suffices to optimally control it, under the resource constraints $\mathscr{C}$, i.e., a model-reduced description of the noisy dynamics. Generally, there will be a trade-off between the model-reduction properties of the frame and the accuracy: a larger frame will lead to a smaller $\varepsilon$, which however necessarily implies that each $\mathcal{I}_{\vec{\alpha} ; \vec{u}, \vec{v}}^{(k)}(T)$ is represented by a sum over a larger domain.

It is clear then why there is a need for a flexible language: one must design $\mathscr{F}$ according to $\mathscr{C}$. The frame language provides a constructive and relatively straightforward mechanism to do so. For instance, one can choose as frame elements a subset of the possible $y_{u, v}^{(\alpha)}(t)$, say $\mathscr{C}_{0}$, and expand every control matrix element in terms of this subset via Eq. (8). If $\mathscr{C}_{0}$ is chosen adequately, the error $\varepsilon$ in the reconstruction can be made small, and an accurate model reduction is achieved. This $\left(\mathscr{F}=\left\{\left.y(t)\right|_{\mathscr{C}_{0}}\right\}\right)$ will be essentially how we build our frames in this paper, as it greatly simplifies the CA-QNS problem: if one chooses as control for a QNS protocol an element of $\mathscr{C}_{0}$, the sum in Eq. (14) can reduce to a single term. Now, while a full CAQNS protocol is not as simplistic as that, this observation gives a glimpse into how much the "correct" language can simplify the task. Note that such a simplification would not be achievable in general if one insists on considering only expansions in orthonormal bases, as the $y_{u, v}^{(\alpha)}(t)$ are typically not orthogonal to each other.

Clearly, our choice for $\mathscr{F}$ is not unique and other ways of building a convenient frame may be possible, depending on the task at hand. In general, this problem is related to that of building a parsimonious model - in the language of the model-reduction literature [44] — which in our context characterizes the ability of the chosen frame to approximate the elements of the control matrix. We leave it to future work to explore what frame, for fixed control capabilities $\mathscr{C}$, allows for maximum parsimony while retaining sufficient predictive power. We stress, however, that the key is tying the choice of $\mathscr{F}$ to the available $\mathscr{C}$ and the tolerance $\varepsilon$. This follows the reasoning that if $\mathscr{C}$ changes then the components of the noise that affect the quantum system also change. Indeed, given a change $\mathscr{C} \mapsto \mathscr{C}^{\prime}$, the first step should be verifying that $\mathscr{F}$ still satisfies the FSF condition to an acceptable $\varepsilon$. Now, the change in $\mathscr{F}$ can take many forms depending on the change in $\mathscr{C}$. It can be as radical as completely changing the functional form of the frame elements or as simple as adding more elements to the original frame. The former is necessary when, for example, the accessible control profiles change from $h_{v}(t) \sim$ $\left.e^{-(t-\tau)^{2} / 2 \sigma^{2}}\right|_{\sigma \in\left[\sigma_{\min }, \sigma_{\max }\right]}$ to $\left.h_{v}^{\prime}(t) \sim e^{-|t-\tau| / 2 \sigma^{2}}\right|_{\sigma \in\left[\sigma_{\min }, \sigma_{\max }\right]}$. In contrast, the latter type of frame change would be sufficient, for example, when the shape of the profiles $h_{v}(t)$ remains the same, but the range of the defining parameters changes, e.g., to $\sigma \in\left[\sigma_{\min }^{\prime}, \sigma_{\max }^{\prime}\right]$.

\section{FRAME-BASED APPROACH TO CHARACTERIZATION AND CONTROL}

\section{A. From noise and control assumptions to frame construction}

We are now ready to deploy our tools. To do so, we first introduce a multiqubit system control problem and 
show that the same frame can be used irrespective of the number of qubits, as long as the control constraints are homogeneous. We exemplify the frame construction for both instantaneous and noninstantaneous control settings and use these in the two key applications we anticipated: QNS beyond the standard frequency domain and the noise-tailored optimized gate design.

\section{The multiqubit model system}

To demonstrate the usefulness of our formalism, we now specialize our system to $N$ qubits and, correspondingly, take $\left\{\Lambda_{u}\right\} \equiv\left\{\Sigma_{u}\right\}$ to be the usual Pauli productoperator basis, with $u \in\left\{0, \ldots, 4^{N}-1\right\}$ and $\Sigma_{0}=\mathbb{I}^{\otimes N}$. The qubits are exposed to additive noise, described as before by $B_{u}^{(a)}(t)=\tilde{B}_{u}^{(a)}(t)+\beta_{u}^{(a)}(t) I_{B}$, along with multiplicative control noise that, for simplicity, we assume to be isotropic, $B_{u}^{(m)}(t) \equiv \beta^{(m)}(t) I_{B}$ for all $u$. We focus on the paradigmatic scenario in which $\mathscr{C}$ comprises $M$ nonoverlapping pulses of duration $\tau \equiv T / M$ applied over $[0, T]$, implemented by

$$
H_{\mathrm{ctrl}}(t)=\left[1+\beta^{(m)}(t)\right] \sum_{j=1}^{M} \theta_{j} h\left(t_{j}, t\right)\left(\vec{n}^{(j)} \cdot \vec{\Sigma}\right) / 2,
$$

where $\vec{\Sigma}$ excludes $\Sigma_{0}, \vec{n}^{(j)} \in \mathbb{R}^{4^{N}-1},\left\|\vec{n}^{(j)}\right\|=1$, and $\theta_{j} \in[0,2 \pi]$ specifies the $j$ th pulse, described by a fixed (normalized) control profile $h\left(t_{j}, t\right)$ centered around $t_{j} \equiv(j-1 / 2) \tau$ and proportional to a window function $W_{j, \tau}(t)$ defined via

$$
W_{j, \tau}(t) \equiv \begin{cases}1, & (j-1) \tau \leq t<j \tau \\ 0, & \text { otherwise }\end{cases}
$$

The above form of $H_{\text {ctrl }}(t)$ is general enough to support single- or multibody Hamiltonian controls, although in a realistic system one would typically be limited to at most two-body Hamiltonians. To simplify our calculations, however, we demand that the possible control inputs $h_{u}(t)$ are the same regardless of $\Sigma_{u}$, that is, the same control constraints apply to any of the possible $H_{\text {ctrl }}(t)$.

Under the above assumptions, one realizes that the form of the frame is independent of the number of qubits, as we will show. Mathematically, the finite support of $h\left(t_{j}, t\right)$ implies that one can write $U_{0}(t)$ as a piecewise function given by

$$
U_{0}(t)=\mathcal{U}_{j} \prod_{\ell=1}^{j-1} U_{\ell}, \quad t \in[(j-1) \tau, j \tau],
$$

where

$$
\begin{gathered}
\mathcal{U}_{j}=e^{-i\left(\theta_{j} / 2\right) \psi_{t}^{(j)} \vec{n}^{(j)} \cdot \vec{\Sigma}}, \quad U_{\ell}=e^{-i\left(\theta_{\ell} / 2\right) \vec{n}^{(\ell) \cdot \vec{\Sigma}}}, \\
\text { with } \quad \psi_{t}^{(j)} \equiv \int_{(j-1) \tau}^{t} d s h\left(t_{j}, s\right) .
\end{gathered}
$$

In turn, this leads to a toggling-frame Hamiltonian [Eq. (3)] of the form

$$
\widetilde{H}(t)=\sum_{u, v}\left[y_{u, v}^{(a)}(t) \Sigma_{v} \otimes B_{u}^{(a)}(t)+y_{v}^{(m)}(t) \beta^{(m)}(t) \Sigma_{v}\right] .
$$

Here, the additive control matrix elements are given by

$$
\begin{aligned}
y_{u, v}^{(a)}(t) & =\frac{1}{2^{N}} \operatorname{Tr}\left[U_{0}(t)^{\dagger} \Sigma_{u} U_{0}(t) \Sigma_{v}\right] \\
& =\frac{1}{2^{N}} \sum_{w=0}^{4^{N}-1} c_{v, w}^{(j-1)} \operatorname{Tr}\left[\mathcal{U}_{j}^{\dagger} \Sigma_{u} \mathcal{U}_{j} \Sigma_{w}\right]
\end{aligned}
$$

for $t \in[(j-1) \tau, j \tau]$, where we have used the fact that

$$
\begin{aligned}
& \left(\prod_{\ell=1}^{j-1} U_{\ell}\right) \Sigma_{v}\left(\prod_{\ell=1}^{j-1} U_{\ell}\right)^{\dagger} \\
& \quad=\sum_{w} \operatorname{Tr}\left[\left(\prod_{\ell} U_{\ell}\right) \Sigma_{v}\left(\prod U_{\ell}\right)^{\dagger} \Sigma_{w}\right] \frac{\Sigma_{w}}{2^{N}} \\
& \quad \equiv \sum_{w} c_{v, w}^{(j-1)} \frac{\Sigma_{w}}{2^{N}} .
\end{aligned}
$$

This ultimately leads to

$$
\begin{aligned}
y_{u, v}^{(a)}(t)=\sum_{w=0}^{4^{m}-1} c_{v, w}^{(j-1)}\{ & k_{j, l ; u, w}^{(0)} \cos \left[\theta_{j} \psi_{t}^{(j)} p_{l}\left(\vec{n}^{(j)}\right)\right] \\
& +k_{j, l ; u, w}^{(1)} \sin \left[\theta_{j} \psi_{t}^{(j)} p_{l}\left(\vec{n}^{(j)}\right)\right] \\
& \left.+k_{j, l ; u, w}^{(2)}\right\},
\end{aligned}
$$

where $\left\{k_{j, l ; u, w}^{(s)}\right\}$ and $p_{l}\left(\vec{n}^{(j)}\right)$ are polynomials of the elements of $\vec{n}^{(j)}$, whose values can be numerically calculated [from Eq. (19)] given a fixed number of qubits $N$.

The multiplicative control matrix elements can be similarly calculated, by noting that $h\left(t_{j}, t\right)$ is assumed to be compactly supported in $[(j-1) \tau, j \tau]$. Thus,

$$
\begin{aligned}
U_{0}(t)^{\dagger} & {\left[h\left(t_{j}, t\right) \vec{n}^{(j)} \cdot \vec{\Sigma}\right] U_{0}(t) } \\
= & h\left(t_{j}, t\right)\left(\prod_{\ell=1}^{j-1} U_{\ell}\right)^{\dagger}\left(\vec{n}^{(j)} \cdot \vec{\Sigma}\right)\left(\prod_{\ell=1}^{j-1} U_{\ell}\right)
\end{aligned}
$$

for $t \in[(j-1) \tau, j \tau]$, since $\mathcal{U}_{j}\left(\vec{n}^{(j)} \cdot \vec{\Sigma}\right) \mathcal{U}_{j}^{\dagger}=\vec{n}^{(j)} \cdot \vec{\Sigma}$. It follows that

$$
y_{v}^{(m)}(t)=\left(\sum_{w} n_{w}^{(j)} c_{v, w}^{(j-1)}\right) h\left(t_{j}, t\right) \theta_{j} / 2 .
$$




\section{The frame}

The important consequence of the above calculations is that $y_{u, v}^{(a)}(t)$ is spanned by the functions $\left\{W_{j, \tau}(t) \sin \left[\eta \psi_{t}^{(j)}\right], W_{j, \tau}(t) \cos \left[\eta \psi_{t}^{(j)}\right]\right\}$ for some $\eta$ and $W_{j, \tau}(t)$, while the $y_{u, v}^{(m)}(t)$ are spanned by the $h\left(t_{j}, t\right)$. Given this, it is then natural to use as frames

$$
\begin{aligned}
& \mathscr{F}_{\#}^{(a)} \equiv\left\{W_{j, \tau} \cos \left[\eta \psi_{t}^{(j)}\right], W_{j, \tau} \sin \left[\eta \psi_{t}^{(j)}\right]\right\}, \\
& \mathscr{F}_{\#}^{(m)} \equiv\left\{h\left(t_{j}, t\right)\right\},
\end{aligned}
$$

for $j \in[1, M]$ and $\eta=2 \pi k / \tilde{N}_{\#}, k \in\left[0, \tilde{N}_{\#}\right]$, where $\tilde{N}_{\#}$ is a free parameter that determines the size of the frame and the tolerance $\varepsilon$, i.e., how well the FSF condition is satisfied. We note that while a finite $\tilde{N}_{\#}$ implies a nonzero $\varepsilon$, the latter decreases as $\tilde{N}_{\#}$ grows: if $\mathscr{F}_{\#}^{(\alpha)}$ contains all admissible $y_{u, v}^{(\alpha)}(t)$, the FSF condition is exactly satisfied $(\varepsilon=0)$. Moreover, the nonoverlapping nature of the pulses implies that the error $\left.\varepsilon\right|_{M}$ for an $M$-pulse control matrix grows only linearly with $M$, i.e., $\left.\varepsilon\right|_{M} \sim \mathcal{O}\left(\left.M \varepsilon\right|_{1}\right)$ for a frame with $N_{\#}=M\left(2 \tilde{N}_{\#}+1\right)$ elements. A larger $\tilde{N}_{\#}$ can thus be chosen so that $\left.\varepsilon\right|_{M}$ is below a user-defined error tolerance as $M$ increases.

The remaining missing elements in our description of the model are the control capabilities $\mathscr{C}$. This is where the frame construction shows its flexibility in dealing with various types of control. For illustration, we consider two scenarios.

(a) First, we address the important limiting case in which control is enacted via perfect, instantaneous pulses. In this case, $\beta^{(m)}(t)=0$ and $h\left(t_{j}, t\right)=$ $\delta(t-j \tau)$, leading to piecewise-constant "switching functions" $\left\{y_{u, v}^{(a)}(t)\right\}$. Given this, $\mathscr{F}_{\#}^{(a)}$ reduces to a collection of window functions $\left\{W_{j, \tau}\right\}_{j=1}^{M}$ for which the FSF condition holds exactly, with $N_{\#}=$ $M$. Thus, in this case, any digital basis suffices as a finite $\mathscr{F}_{\#}^{(a)}$, and an especially compelling choice is provided by the Walsh functions, thanks to their potential for minimizing sequencing complexity [45-47].

(b) Second, we consider a windowed Gaussian control profile, $h\left(t_{j}, t\right)=W_{j, \tau} e^{-\left(t-t_{j}\right)^{2} / 2 \sigma^{2}}$ with $\sigma=1 \mu \mathrm{s}$, $\tau=10 \mu \mathrm{s}$, although other possibilities, such as square or Slepian pulses [20], can be easily accommodated. With $M=1$ and $\mathscr{F}_{\#}^{(a, m)}$ as above, one finds that $\left.\varepsilon\right|_{\tilde{N}_{\#}=2}=2.4 \times 10^{-5}$, i.e., a $1.1 \%$ relative error [48], whereas $\left.\varepsilon\right|_{\tilde{N}_{\#=4}}=2.8 \times 10^{-8}$, i.e., a $0.0014 \%$ relative error. That is, a modest-size frame ensures that the FSF condition is basically satisfied. Exemplifying the $M$ and $\tilde{N}_{\#}$ interplay, for $M=100$ pulses, a value of $\tilde{N}_{\#}=4$, i.e., an $N_{\#}=$ 900 elements frame, ensures an overall error of approximately $0.15 \%$.
The above highlights the aforementioned trade-off between model reduction and accuracy, highlighting the importance of building a (maximally) parsimonious frame - our choice here need not be optimal as it is only meant as a proof of principle. Moreover, we note that the dynamics of the system generally depends on linear combinations of the $\mathcal{I}_{\vec{\alpha}: \vec{u}, \vec{v}}^{(k)}\left(k \leq k_{\max }\right)$, where the number of terms is typically a function of the range of the values the indices $\{u, v, \alpha\}$ can take. Accordingly, one must ensure that the error in each $\mathcal{I}_{\vec{\alpha} ; \vec{u}, \vec{v}}^{(k)}$ and also in the relevant linear combinations is small. Ultimately, this implies that, while the form of the frame is the same regardless of the dimension of the system $d$, the number of nonoverlapping pulses $M$, or the largest order of perturbation being considered $k_{\max }$, the tolerance $\varepsilon$ must be small enough — and thus $\tilde{N}_{\#}$ must be large enough—so that the overall error is below a user-defined tolerance for a given $d, M$, and $k_{\max }$.

\section{Case study: single-qubit reduced dynamics}

The final step is to obtain the expectation values of system's observables, $E[O(t)]_{\rho_{S} \otimes \rho_{B}}$. To simplify our expressions and the analysis in the illustrative applications, we specialize in what follows to the simplest paradigmatic setting of a single-qubit dephasing model. Thus, we consider additive noise only along one direction, $B_{u}(t) \equiv B_{z}(t)$, whereas the multiplicative noise will be present, as in Eq. (18), whenever the control is assumed to be imperfect. Noting that in this scenario the $\Sigma_{u}$ reduce to the single-qubit Pauli operators $\sigma_{u}, u \in\{0, x, y, z\}$, Eq. (20) simplifies to

$$
\begin{aligned}
y_{u}^{(a)}(t) \equiv y_{z, u}^{(a)}(t)= & \sum_{v} c_{u, v}^{(j-1)}\left(k_{j, v}^{(0)} \cos \left[\theta_{j} \psi_{t}^{(j)}\right]\right. \\
& \left.+k_{j, v}^{(1)} \sin \left[\theta_{j} \psi_{t}^{(j)}\right]+k_{j, v}^{(2)}\right) .
\end{aligned}
$$

Furthermore, we enforce in our model that the multiplicative and additive noise sources are uncorrelated, i.e., $\left\langle B^{(m)}\left(t_{1}\right) B^{(a)}\left(t_{2}\right)\right\rangle=\left\langle B^{(m)}\left(t_{1}\right)\right\rangle\left\langle B^{(a)}\left(t_{2}\right)\right\rangle=0$. We do not, however, require stationarity nor Gaussianity.

In a suitable weak-coupling regime where, formally, $\max _{\left\{t_{i}\right\}}\left\langle B^{(\alpha)}\left(t_{1}\right) \cdots B^{(\alpha)}\left(t_{k}\right)\right\rangle T^{2} \ll 1$, Eq. (4) becomes

$$
E[O(T)]_{\rho_{S} \otimes \rho_{B}} \approx\left\langle\operatorname{Tr}_{S}\left\{\left[I_{S}-D_{O}^{(2)}(T)\right] \rho_{S} \widetilde{O}(T)\right\}\right\rangle,
$$

where the second-order Dyson term $D_{O}^{(2)}(T)$ can be written as a functional of a reduced set of CA spectra. Specifically, the components of the spectra relevant to $\mathscr{C}$ are found to be (see Appendix C for full detail)

$$
\left.\overline{\mathscr{S}}\right|_{\mathscr{C}}=\left\{\bar{S}_{\alpha}^{(+)}\left(n, n^{\prime}\right),\left[\bar{S}_{\alpha}^{(-)}\left(n, n^{\prime}\right)-\bar{S}_{\alpha}^{(-)}\left(n^{\prime}, n\right)\right]\right\}
$$

for $n \in\left[1, N_{\#}\right]$ and $\alpha \in\{a, m\}$, where $\bar{S}_{\alpha}^{( \pm)}\left(n, n^{\prime}\right)$ are associated with the "classical" $(+)$ and "quantum" $(-)$ two-point 
bath correlation functions [18],

$$
C_{ \pm}^{(\alpha)}\left(t_{1}, t_{2}\right) \equiv\left\langle\left[B^{(\alpha)}\left(t_{1}\right), B^{(\alpha)}\left(t_{2}\right)\right]_{ \pm}\right\rangle
$$

with $\left[A, A^{\prime}\right]_{ \pm} \equiv A A^{\prime} \pm A^{\prime} A$. Since only FFs $\left\{F_{\alpha ; u}^{(1)}(n, T)\right\}$ allowed by $\mathscr{C}$ can be generated, only the above noise parameters can be inferred from the reduced dynamics via CA QNS. Still, such finite information suffices for the prediction - and eventual optimization - of the qubit dynamics at time $T$ under any of the (infinite) control sequences allowed by $\mathscr{C}$.

\section{B. QNS beyond the frequency domain}

Regardless of whether multipulse or continuous control modulation is employed (such as, respectively, in combbased [19] or Slepian- [20] and spin-locking-based [15,21] protocols), existing QNS methods largely rely on the possibility to describe the noise properties in the frequency domain. This prevents applicability to nonstationary noise $[27,28]$ as well as noise with singular correlation functions [49], which must be described in the time domain.

To illustrate how such limitations are overcome in our frame-based approach, in this section we implement CA QNS via instantaneous perfect pulses applied at separate uniform intervals over a total time $T$. We thus specialize the control Hamiltonian in Eqs. (16) and (17) to

$$
H_{\mathrm{ctrl}}(t)=\sum_{j=1}^{M} \delta(t-j \tau) \theta_{j} \vec{n}^{(j)} \cdot \vec{\sigma} / 2,
$$

where $M$ is now the number of intervals and $\tau=T / M$ is the minimum separation time between pulses (that is, each pulse is applied at a nonzero multiple of the minimum "switching time" $\tau>0$ [50]). A direct specialization of Eq. (23) reveals that the control matrix elements (i) are necessarily linear combinations of $\left\{W_{t_{j}, \tau} \cos \left[\theta_{j} \psi_{t}^{(j)}\right], W_{t_{j}, \tau} \sin \left[\theta_{j} \psi_{t}^{(j)}\right]\right\}$ and (ii) satisfy the constraints $y_{u}^{(a)}(t) \in[-1,1]$; and $\sum_{u}\left|y_{u}^{(a)}(t)\right|^{2}=1$. As discussed in Sec. IV A, a suitable (self-dual) frame in this case is the Walsh basis, which obeys the FSF condition exactly $(\varepsilon=0)$. In our CA-QNS protocol, we chose $\theta_{j} \in$ $\{0, \pi / 2, \pi\}$ and $\vec{n}^{(j)}=(0,1,0)$ for all $j$. In contrast with previous Walsh-based characterization methods [47], the use of non- $\pi$ pulses now makes it possible to generate control matrix elements $\left\{y_{u}^{(a)}(t)\right\}$ that are linear combinations of Walsh functions with $M$ switches over the time range $[0, T]$. This leads to the ability to infer the Walsh-basis CA spectra $\bar{S}_{\alpha}^{( \pm)}\left(n, n^{\prime}\right)$ for all $n, n^{\prime}$, not only generalizing the approach of Ref. [47] beyond reconstruction of signals with a finite number of frequency components, but allowing reconstruction of nonstationary noise.

In particular, we apply the CA-QNS protocol to two distinct settings: (i) a classical nonstationary Wiener process and (ii) a genuinely quantum (bosonic) nonstationary environment. Our task will be to show that the protocol provides sufficient information about the correlation functions, which in turns allows one to infer the parameters describing the noise model under consideration. Full details about the sequences we used are included in Appendix C 2.

\section{Example 1. Nonstationary noise from a classical time-dependent diffusion process}

Motivated by the physical setting described in Ref. [27], we consider noise induced by a random walk of molecules in solution, resulting in translational diffusion in the presence of an external magnetic field - a process ubiquitously encountered in liquid-state NMR and beyond. The relevant dephasing Hamiltonian may be written as

$$
H(t)=\sigma_{z} \cdot \hbar \gamma_{M} G \beta(t) \equiv \sigma_{z} B(t),
$$

where $\gamma_{M}=2.67 \times 10^{8} \mathrm{rad} \mathrm{s}^{-1} \mathrm{~T}^{-1}$ is the gyromagnetic ratio of protons, $G=0.0214 \mathrm{~T} \mathrm{~m}^{-1}$ is a constant magnetic gradient along $z$, and $\beta(t)$ is a Gaussian stochastic process representing the Brownian excursions of the molecule. That is,

$$
\langle\beta(t)\rangle=0, \quad C\left(t_{1}, t_{2}\right) \equiv\left\langle\beta\left(t_{1}\right) \beta\left(t_{2}\right)\right\rangle=D \min \left(t_{1}, t_{2}\right),
$$

with $D$ being the molecular diffusion constant. While the nonstationary nature of the process is evident $-C\left(t_{1}, t_{2}\right)$ is not invariant under an arbitrary shift in time-we further assume here that the diffusion constant varies periodically in time according to $D \mapsto D \cos \left(v t_{1}\right) \cos \left(v t_{2}\right)$, where $v$ is an unknown angular frequency [see Fig. 1(a)]. This renders the modified process $\beta(t)$ second-order cyclostationary [51], with

$$
\begin{aligned}
& C\left(t_{1}+2 \pi n / v, t_{2}+2 \pi n / v\right) \\
& \quad=C\left(t_{1}, t_{2}\right) \quad \text { for all } t_{1}, t_{2}, n \in \mathbb{Z} .
\end{aligned}
$$

We further assume that, for each of the control experiments required by a QNS protocol, the system is reinitialized, i.e., the diffusion process is also effectively reset.

Applying the CA-QNS protocol, we estimate the CA spectra, $\bar{S}^{(+)}\left(n, n^{\prime}\right)$ [note that in this case $\bar{S}^{(-)}\left(n, n^{\prime}\right)=0$ ], and, from there, the digitized correlation function $\left.\left\langle\left[B\left(t_{1}\right), B\left(t_{2}\right)\right]_{+}\right\rangle\right|_{\mathscr{C}}$ by using the general relation

$$
\begin{aligned}
& \left.\left\langle\left[B\left(t_{1}\right), B\left(t_{2}\right)\right]_{ \pm}\right\rangle\right|_{\mathscr{C}} \\
& \quad=\sum_{n, n^{\prime}}\left[\bar{S}^{( \pm)}\left(n, n^{\prime}\right) \pm \bar{S}^{( \pm)}\left(n^{\prime}, n\right)\right] \tilde{\phi}_{n}\left(t_{1}\right) \tilde{\phi}_{n^{\prime}}\left(t_{2}\right) .
\end{aligned}
$$

The result of the digital reconstruction is shown in Fig. 1. In addition, by leveraging the knowledge of the physical origin of the noise, we infer the parameters in the model Hamiltonian, $P \equiv\{D, v\}$ (see Table II), by following an 

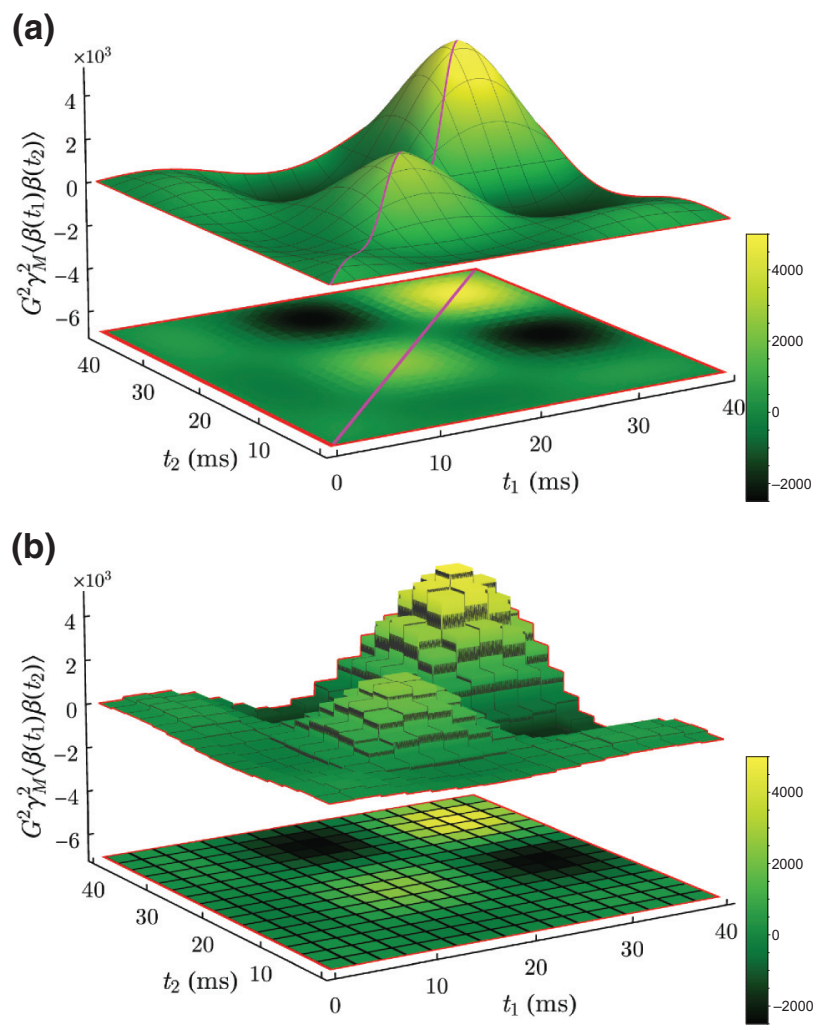

FIG. 1. (a) Theoretical versus (b) digitally reconstructed version of the nonstationary correlation function $\left\langle\beta\left(t_{1}\right) \beta\left(t_{2}\right)\right\rangle$ in units of $\operatorname{rad}^{2} \mathrm{~s}^{-2}$ for a time resolution of $T / M, T=40 \mathrm{~ms}$ and $M=16$. The solid magenta curve in (a) highlights where $\left\langle\beta\left(t_{1}\right) \beta\left(t_{2}\right)\right\rangle$ is not differentiable.

approach we outline in more detail in Example 2 below. It should be noted that the accuracy in the estimation of the parameters relies on the control capabilities. While in our example the given $T=40 \mathrm{~ms}$ and $M=16$ suffice to achieve a good estimation, this need not be the case in general, as we exemplify next.

\section{Example 2. Nonstationary noise from a quantum time-dependent bosonic environment}

Consider now a scenario where the qubit couples to a two-mode bosonic environment via a periodically varying coupling operator, that is,

$$
B(t)=\sum_{\ell=1}^{2} g_{\ell}(t)\left(e^{i \Omega_{\ell} t} a_{\ell}^{\dagger}+\text { H.c. }\right), \quad g_{\ell}(t)=\bar{g}_{\ell} \cos \left(\mathrm{w}_{\ell} t\right) .
$$

TABLE II. Actual and estimated parameters for the classical oscillatory-diffusive model of Example 1.

\begin{tabular}{lcc}
\hline \hline & $D\left(\mathrm{~m}^{2} \mathrm{~s}^{-1}\right)$ & $v(\mathrm{~Hz})$ \\
\hline Actual & $2.3 \times 10^{-9}$ & $60 \pi \approx 188.496$ \\
Estimated & $2.3 \times 10^{-9}$ & 188.496 \\
\hline \hline
\end{tabular}

Again, the time dependence in the couplings makes the noise nonstationary, as $\left\langle B\left(t_{1}\right) B\left(t_{2}\right)\right\rangle$ is manifestly a function of both $t_{1}+t_{2}$ and $t_{1}-t_{2}$, although periodic in the former. Also, we assume that the initial state of the bath is thermal, $\rho_{B} \propto e^{-\sum_{\ell=1}^{2} \beta_{\ell} \hbar \Omega_{\ell} a_{\ell}^{\dagger} a_{\ell}}$ with $\beta_{\ell} \equiv 1 / k_{B} T_{\ell}$, so that $\langle B(t)\rangle_{c}=0$, and the symmetric and antisymmetric parts of the correlation function can be respectively written as

$$
\begin{aligned}
\left\langle\left[B\left(t_{1}\right), B\left(t_{2}\right)\right]_{+}\right\rangle_{c}= & \sum_{\ell}\left|\bar{g}_{\ell}\right|^{2}\left\{\cos \left[w_{\ell}\left(t_{1}+t_{2}\right)\right]\right. \\
& \left.+\cos \left[w_{\ell}\left(t_{1}-t_{2}\right)\right]\right\} \cos \left[\Omega_{\ell}\left(t_{1}-t_{2}\right)\right] \\
& \times \operatorname{coth}\left(\hbar \beta_{\ell} \Omega_{\ell} / 2\right), \\
\left\langle\left[B\left(t_{1}\right), B\left(t_{2}\right)\right]_{-}\right\rangle_{c}= & -i \sum_{\ell}\left|\bar{g}_{\ell}\right|^{2}\left\{\cos \left[w_{\ell}\left(t_{1}+t_{2}\right)\right]\right. \\
& \left.+\cos \left[w_{\ell}\left(t_{1}-t_{2}\right)\right]\right\} \sin \left[\Omega_{\ell}\left(t_{1}-t_{2}\right)\right] .
\end{aligned}
$$

Applying the CA-QNS protocol described earlier, one can infer $\bar{S}^{(+)}\left(n, n^{\prime}\right)$ and $\bar{S}^{(-)}\left(n, n^{\prime}\right)-\bar{S}^{(-)}\left(n^{\prime}, n\right)$, and, from there, obtain a digital reconstruction [by using Eq. (24)] of both the classical and quantum components of the correlation function. While this information is also crucial for control, in this section we focus only on the open-system characterization aspect of our problem, i.e., leveraging the information CA QNS provides and knowledge of the noise model to estimate the relevant parameters. We execute the protocol for two resolutions $\tau=T / M$, namely, for $T=1536 \mathrm{ps}, M=16$ and $T=16 \mathrm{ps}, M=16$. The resulting reconstructions are presented in Figs. 2 and 3, respectively, which reveal the impact of the time resolution. As Fig. 2 demonstrates, the coarse resolution reconstruction does not detect the effect of the fast oscillations. Equipped only with this information, it is not possible to infer the value of comparatively large frequencies with high accuracy. In contrast, the high-resolution reconstruction - consistent with a minimum interpulse timing of $1 \mathrm{ps}$ (see Fig. 3) - can detect the fast oscillations in our model, and allows us to accurately estimate all the model parameters. By using both the low and high resolution, we infer the physical parameters as follows. The parameters of interest are the set

$$
P \equiv\left\{w_{1}, w_{2}, \Omega_{1}, \Omega_{2}, \bar{g}_{1}, \bar{g}_{2}, T_{1}, T_{2}\right\}
$$

Assuming knowledge of the model, we estimate them by minimizing a cost function

$$
\mathcal{C}_{\$}(P) \equiv \sum_{\mu= \pm} \sum_{n, n^{\prime}=1}^{N_{\#}}\left[\left.S^{(\mu)}\left(n, n^{\prime}\right)\right|_{P}-\hat{S}^{(\mu)}\left(n, n^{\prime}\right)\right]^{2}
$$

where the $\left.S^{( \pm)}\left(n, n^{\prime}\right)\right|_{P}$ is calculated from the assumed model for a given set of parameters $P$, and $\hat{S}^{( \pm)}\left(n, n^{\prime}\right)$ 
(a)

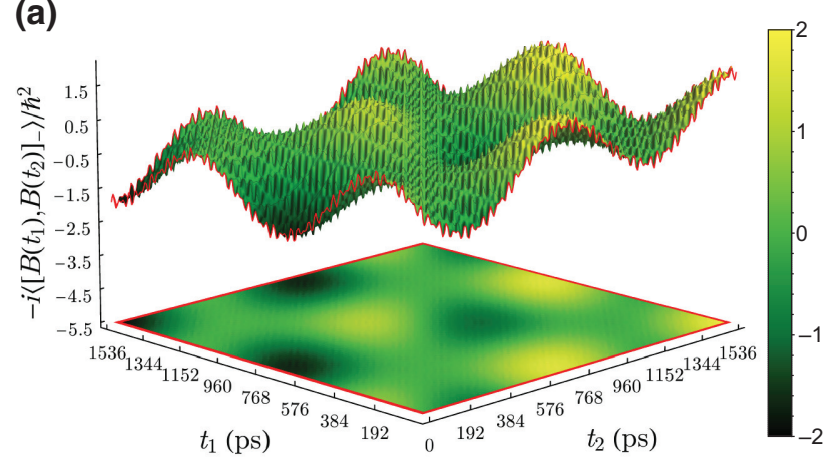

(c)

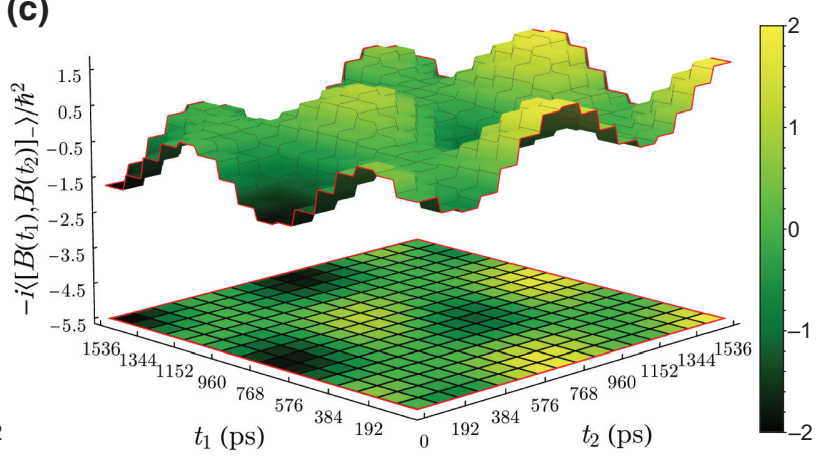

(b)

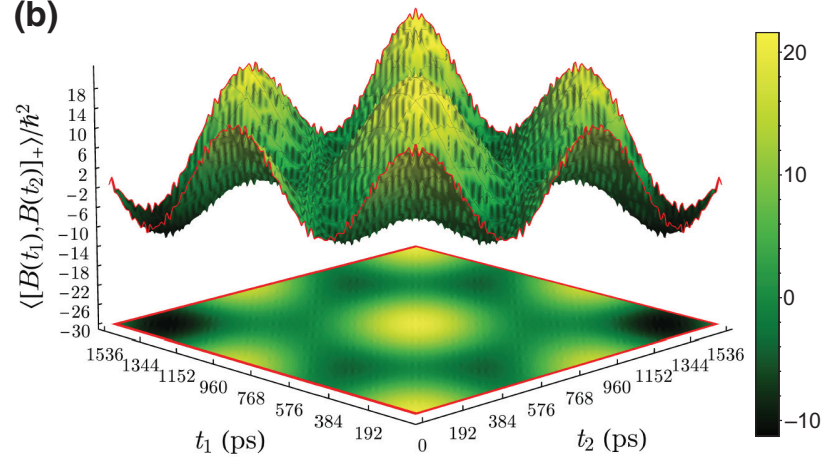

(d)

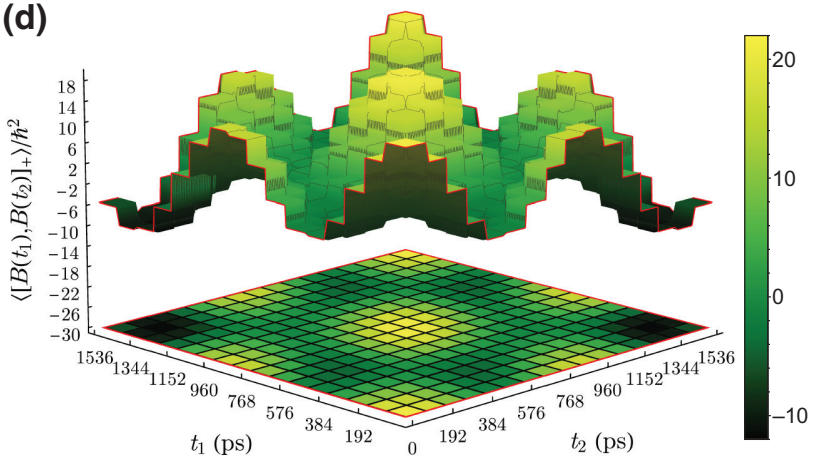

FIG. 2. (a) Actual antisymmetric (quantum) and (b) symmetric (classical) components of the correlation function, and their corresponding digital reconstructions [(c) and (d), respectively] in units of $10^{18} \mathrm{~Hz}^{2}$ for $T=1536 \mathrm{ps}$ and $M=16$. In our numerical simulations we choose: $w_{1}=125 \pi / 96 \mathrm{GHz}, w_{2}=7.5 w_{1}, \Omega_{2}=60 \Omega_{1}, \Omega_{1}=125 \pi / 128 \mathrm{GHz}$, couplings $\bar{g}_{1} / \hbar=976.56 \mathrm{MHz}$, $\bar{g}_{2} / \hbar=345.27 \mathrm{MHz}$, and inverse temperatures $\hbar \beta_{1}=61.44 \mathrm{ps}, \hbar \beta_{2}=2.05 \mathrm{ps}$ (corresponding to $T_{1}=0.12 \mathrm{~K}$ and $T_{2}=3.73 \mathrm{~K}$ ). The reconstruction with the chosen resolution is capable of capturing the slow oscillations, but not the fast oscillations.

is estimated as $\hat{S}^{( \pm)}\left(n, n^{\prime}\right)=\bar{S}^{( \pm)}\left(n, n^{\prime}\right) \pm \bar{S}^{( \pm)}\left(n^{\prime}, n\right)$, with the input spectra calculated from the $\overline{\mathscr{S}}_{\mathscr{C}}$.

We perform the optimization, $\operatorname{argmin}_{P} \mathcal{C}_{\$}(P)$, in two settings: (i) with only the low (or coarse) resolution $\overline{\mathscr{S}}_{\mathscr{C}}$ and (ii) combining both the low- and high-resolution information. As expected, the optimization in the first approach only accurately estimates the parameters corresponding to the slow frequencies, but not the high frequency generating the fast oscillations in the correlation functions. In contrast, in the second more powerful approach, we estimate all the parameters of interest with high accuracy (assuming no other source of error but the digitization of the reconstruction induced by the available control). We summarize our estimation results in Table III. We highlight that the example above shows that it is possible to perform "local bath thermometry" using a single-qubit probe in a "short-time" regime, in contrast with existing approaches for stationary noise, which require either a steady-state regime [52] or multiple probes [18].

\section{Control-adapted noise-tailored optimized gate design}

Beyond the task of bath characterization, and perhaps more relevant to the implementation of quantum technologies, one can leverage the information QNS provides to achieve high-fidelity operations, by tailoring the control to the noise affecting the qubit, via numerical optimal control algorithms [8,9] or geometric techniques [53]. While the details are method dependent, and the underlying non-Markovian dynamics may be modeled in different forms (i.e., via a master equation or a Dyson expansion), a common feature is that - in the absence of extra assumptions on the bath - information about the noise correlation functions $\left\{\left\langle B_{v_{1}}^{\left(\alpha_{1}\right)}\left(t_{\mu(1)}\right) \cdots B_{v_{k}}^{\left(\alpha_{k}\right)}\left(t_{\mu(k)}\right)\right\rangle\right\}$ is needed as an input.

The issue, however, is that, given limited control capabilities $\mathscr{C}$, one cannot characterize such correlation functions in full, but rather only the portion of them that is relevant to $\mathscr{C}$. While the intuition rings true, namely, one can only infer what the control allows one to see, this is also evident from our previous developments. Take, for example, the instantaneous pulse case, for which $\varepsilon=0$; hence, there is no loss of information due to model reduction. Given a minimum-switching time constraint, we showed that one can only recover a digitized version of the true correlation functions, $\left\langle\left.\left[B\left(t_{1}\right), B\left(t_{2}\right)\right]_{ \pm}\right|_{\overline{\mathscr{F}}}\right\rangle$. The key point, however, is that, while $\left\langle\left[B\left(t_{1}\right), B\left(t_{2}\right)\right]_{ \pm} \mid \overline{\mathscr{F}}\right\rangle$ is only a 

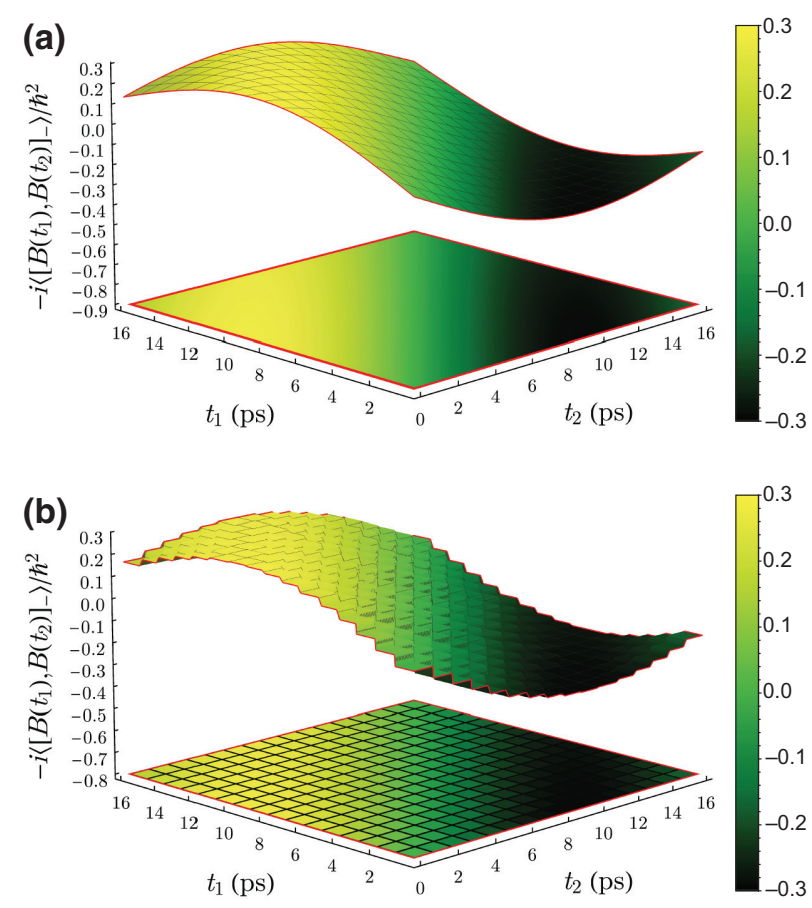

FIG. 3. (a) Actual antisymmetric (quantum) component of the correlation function and (b) its corresponding digital reconstruction in units of $\left(10^{9} \mathrm{GHz}\right)^{2}$ for $T=16 \mathrm{ps}$ and $M=16$. While the resolution is $\tau=1 \mathrm{ps}=16 \mathrm{ps} / 16=1536 \mathrm{ps} / 1536$, equivalent to performing a digital reconstruction on a total time of 1536 ps with a $1536 \times 1536$ grid, note that in the reconstruction with $T=16 \mathrm{ps}$, the slow oscillations cannot be appreciated.

"control-reduced" version of $\left[B\left(t_{1}\right), B\left(t_{2}\right)\right]$, it is exactly what is needed to predict, and eventually optimize, the dynamics of any pulse sequence resulting from $\mathscr{C}$.

To demonstrate this, we consider the task of executing a target quantum gate $G$ with the highest possible fidelity. Importantly, we assume no a priori knowledge of the noise correlation functions and, in contrast to the previous subsection, we consider the realistic setting of noisy bounded-strength (noninstantaneous) control. Specifically, we restrict our control capabilities $\mathscr{C}$ to the scenario where $h_{u}(t)$ in each of the $M=2$ pulses has a Gaussian shape, and a total execution time $T=10 \mu \mathrm{s}$. In the non-Markovian setting, achieving a high-quality operation implies minimizing an appropriate cost function, which is a functional of the overlap integrals $\mathcal{I}_{\vec{\alpha} ; \vec{u}, \vec{v}}^{(k)}(T)$ and whose explicit form depends on the perturbative expansion of choice. While many choices are possible, we define ours as follows. Noting that a single-qubit gate $G$ can be specified by the expectations

$$
E\left[\sigma_{u}\right]_{\sigma_{v}}=\operatorname{Tr}_{S}\left[G \sigma_{v} G^{\dagger} \sigma_{u}\right] \equiv E_{u, v ; \mathrm{G}}, \quad u, v \in\{0, x, y, z\}
$$

we define the cost function for executing $G$ over time $T$ as

$$
\mathscr{E}_{\$}^{G}(P ; T) \equiv \sum_{u, v}\left|E_{u, v ; G}-e_{u, v}(P ; T)\right|^{2}
$$

where $e_{u, v}(P ; T)$ is a fixed-order (here, $\left.k=2\right)$ perturbative expansion of $E\left[\sigma_{u}(T)\right]_{\sigma_{v} \otimes \rho_{B}}$ corresponding to a control parameter set $P=\left\{\theta_{i}, \vec{n}^{(i)}\right\}$, calculated using full knowledge of $\left\langle\left[B\left(t_{1}\right), B\left(t_{2}\right)\right]_{+}\right\rangle$(as would be the case in numerical optimal control routines). In contrast, when we specialize our equations to the model-reduced representation associated with $\mathscr{F}$ of the integrals $\mathcal{I}_{\vec{\alpha} ; \vec{u}, \vec{v}}^{(k)}(T)$ [such as, e.g., Eq. (14)], we write the cost function as

$$
\left.\left.\mathscr{E}_{\$}^{G}(P ; T)\right|_{\mathscr{F}} \equiv \sum_{u, v}\left|E_{u, v ; G}-e_{u, v}(P ; T)\right|_{\mathscr{F}}\right|^{2} .
$$

In each case the optimization, given $\mathscr{C}$, consists in finding the set $P$ such that the corresponding cost function is minimized. Our objective will be to show that the modelreduced and full-knowledge optimal solutions, namely,

$$
\begin{aligned}
\left.P^{*}\right|_{\mathscr{F}} & \left.\equiv \operatorname{argmin}_{P} \mathscr{E}_{\mathscr{S}} G(P ; T)\right|_{\mathscr{F}} \quad \text { versus } \\
P^{*} & \equiv \operatorname{argmin}_{P} \mathscr{E}_{\mathscr{S}} G(P ; T),
\end{aligned}
$$

yield similar performances, in the sense that $\mathscr{E}_{\mathbb{S}}^{G}\left(\left.P^{*}\right|_{\mathscr{F}} ; T\right)$ $\approx \mathscr{E}_{S}^{G}\left(P^{*} ; T\right)$. If this indeed happens, it follows that there is no significant loss of information and effective model reduction has been achieved, and we show in the case below.

\section{Noise characterization}

Since we stipulated that no knowledge of the noise correlation functions is available, we first need to characterize the open quantum system to the best of our ability, i.e., within the limits allowed by $\mathscr{C}$. As in Sec. IV A 3 , we assume that the qubit is evolving in the presence of uncorrelated additive and multiplicative noise sources

TABLE III. Actual and estimated physical parameters in the two-mode bosonic model described by Eqs. (25a) and (25b). The second row shows the actual parameters of the model. The third and the forth rows show the estimated parameter using CA spectra from coarse reconstruction only and both coarse and fine reconstruction, respectively.

\begin{tabular}{lcccccccc}
\hline \hline & $\bar{g}_{1} / \hbar(\mathrm{MHz})$ & $\bar{g}_{2} / \hbar(\mathrm{MHz})$ & $\Omega_{1}(\mathrm{GHz})$ & $\Omega_{2}(\mathrm{GHz})$ & $w_{1}(\mathrm{GHz})$ & $w_{2}(\mathrm{GHz})$ & $\hbar /\left(k_{B} T_{1}\right)(\mathrm{ps})$ & $\hbar /\left(k_{B} T_{2}\right)(\mathrm{ps})$ \\
\hline Actual & 976.56 & 345.27 & 3.07 & 184.08 & 4.09 & 30.68 & 61.44 & 2.05 \\
Coarse & 976.55 & 317.80 & 3.07 & 179.63 & 4.09 & 25.93 & 61.44 & 2.03 \\
Coarse and fine & 976.56 & 345.27 & 3.07 & 184.08 & 4.09 & 30.68 & 61.44 & 2.05 \\
\hline \hline
\end{tabular}



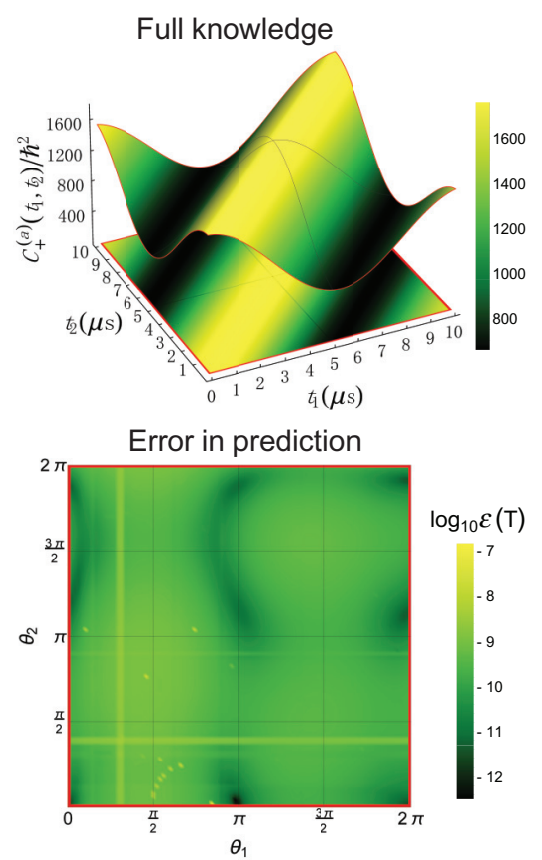
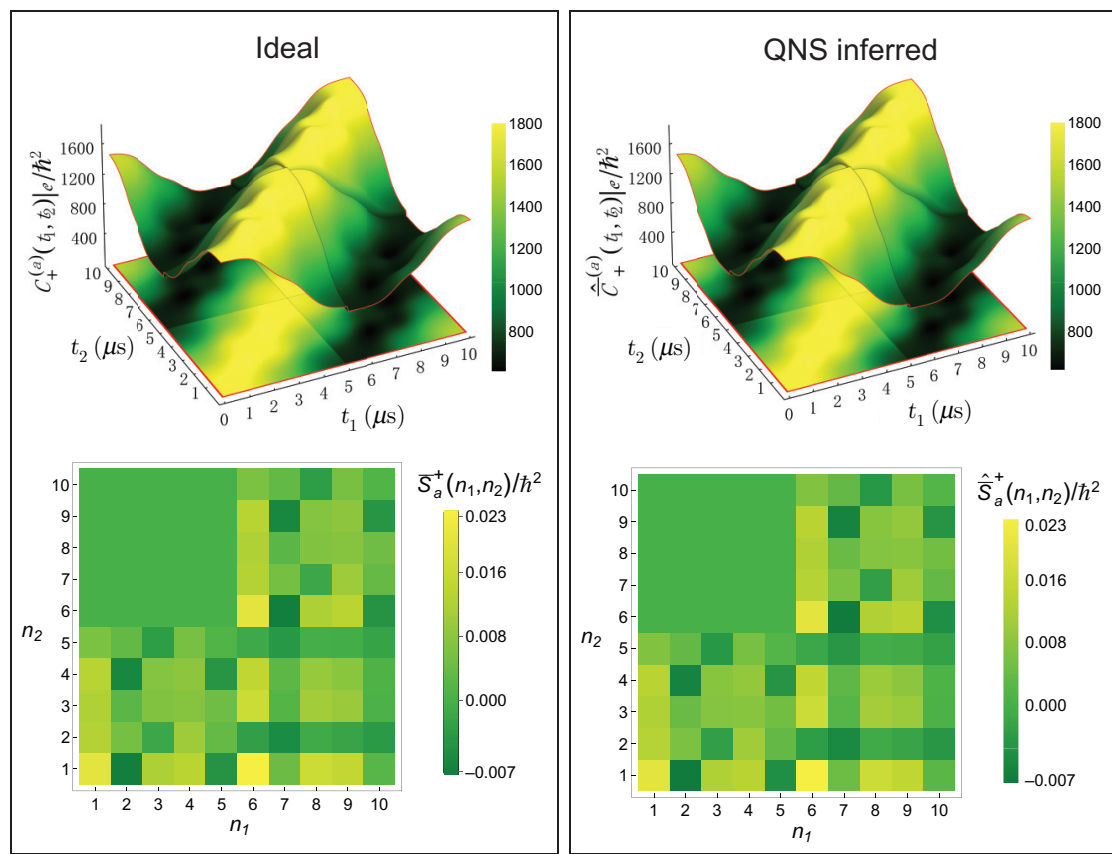

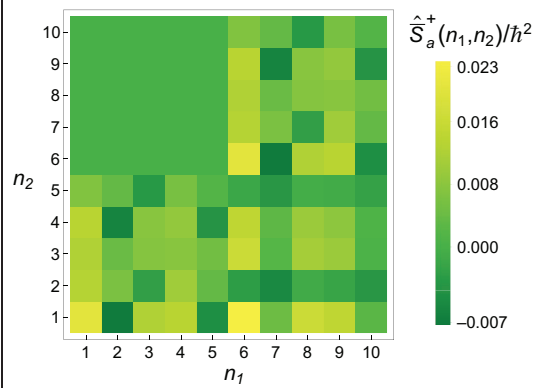

FIG. 4. The model assumes a correlation function (upper left) $C_{+}^{(a)}\left(t_{1}-t_{2}\right)=\left\langle\left[B^{(a)}\left(t_{1}\right), B^{(a)}\left(t_{2}\right)\right]_{+}\right\rangle$unknown to the experimenter. Given $\mathscr{C}$ (hence $\mathscr{F}$ ), one can calculate the ideal frame projection $\bar{S}_{a}^{+}\left(n_{1}, n_{2}\right)$ (bottom middle) and the corresponding time-domain representation $\left[B^{(a)}\left(t_{1}\right), B^{(a)}\left(t_{2}\right)\right]_{+} \mid \mathscr{F}$ (top middle). A CA-QNS protocol provides an accurate estimate $\hat{\bar{S}}_{a}^{+}\left(n_{1}, n_{2}\right) / \hbar^{2}$ (bottom right) of $\bar{S}_{a}^{+}\left(n_{1}, n_{2}\right) / \hbar^{2}$, with a maximum absolute error of $5.38 \times 10^{-4}$. For multiplicative noise, our estimates are $\hat{\bar{S}}_{m}^{+}(1,1) / \hbar^{2}=1.125 \times$ $10^{-3} \mathrm{~Hz}^{2}$ and $\hat{\bar{S}}_{m}^{+}(2,1) / \hbar^{2}=2.251 \times 10^{-3} \mathrm{~Hz}^{2}$, with relative errors less than $0.001 \%$. The magnitude of these errors depends on a nonzero $\varepsilon$. The distance $\mathscr{E}(P ; T)$ between the actual dynamics — calculated using $\left[B^{(\alpha)}\left(t_{1}\right), B^{(\alpha)}\left(t_{2}\right)\right]_{+}$- and the predicted dynamics — using $\hat{\bar{S}}_{\alpha}^{+}\left(n_{1}, n_{2}\right)$ —at the time $T$ is small. We showcase the prediction error by randomly selecting pulse directions [here we use $\vec{n}^{(1)}=(0.658,0.751,-0.052)$ and $\left.\vec{n}^{(2)}=(-0.411,0.000,0.912)\right]$ and sweeping over all pulse angles allowed by $\mathscr{C}$ (bottom left). All figures are given in units of $\mathrm{kHz}^{2}$.

(both zero mean), which we take here to be stationary and characterized by correlation functions $C_{+}^{(\alpha)}\left(t_{1}-t_{2}\right)$, $\alpha=a, m$. While unknown to the experimenter, for demonstration, we choose the latter to be the inverse Fourier transforms of

$$
\begin{aligned}
& S^{(a)}(\omega)=\frac{b_{0}^{(a)}}{1+c_{0}^{(a)} \omega^{2}}+\frac{b_{1}^{(a)}}{1+c_{1}^{(a)}\left(\omega-\omega_{1}^{(a)}\right)^{2}} \\
& S^{(m)}(\omega)=b_{0}^{(m)} e^{-\left(\omega-\omega_{0}^{(m)}\right)^{2} / 2 c_{0}^{(m)}},
\end{aligned}
$$

with parameters $b_{0}^{(a)} / \hbar=400 \mathrm{kHz}, c_{0}^{(a)}=0.004 \mathrm{~ms}^{2}$, $b_{1}^{(a)} / \hbar=10^{6} \mathrm{kHz}, \quad c_{1}^{(a)}=0.64 \mathrm{~s}^{2}, \quad \omega_{1}^{(a)}=600 \mathrm{kHz}$, $b_{0}^{(m)} / \hbar=2 \mathrm{mHz}, c_{0}^{(m)} / \hbar=5 \sqrt{2 \pi} \mathrm{Hz}$, and $\omega_{0}^{(m)}=50 \mathrm{~Hz}$. The total time $T$ is chosen so that the weak-coupling approximation is valid, allowing the cumulant or Dyson expansion to be truncated at order two. We also assume that we can prepare any Pauli eigenstate at $t=0$ and measure any Pauli observable at times $t=T / 2$ and $t=T$.
Recalling our declared $\mathscr{C}$, i.e., $M=2$ pulses and $\tilde{N}_{\#}=$ 2 , we start by building the relevant frames. Namely,

$$
\begin{aligned}
\mathscr{F}_{\#}^{(a)}=\left\{\phi_{n}^{(a)}\right\} \equiv\{ & W_{1, T / 2}, W_{2, T / 2}, \\
& W_{1, T / 2} \cos \left[\pi \psi_{t}^{(1)}\right], W_{1, T / 2} \sin \left[\pi \psi_{t}^{(1)}\right], \\
& W_{2, T / 2} \cos \left[\pi \psi_{t}^{(2)}\right], W_{2, T / 2} \sin \left[\pi \psi_{t}^{(2)}\right], \\
& W_{1, T / 2} \cos \left[2 \pi \psi_{t}^{(1)}\right], W_{1, T / 2} \sin \left[2 \pi \psi_{t}^{(1)}\right], \\
& \left.W_{2, T / 2} \cos \left[2 \pi \psi_{t}^{(2)}\right], W_{2, T / 2} \sin \left[2 \pi \psi_{t}^{(2)}\right]\right\}
\end{aligned}
$$

(see also Fig. 8 in Appendix D) and

$$
\mathscr{F}_{\#}^{(m)}=\left\{\phi_{n}^{(m)}\right\} \equiv\left\{h\left(t_{1}, t\right), h\left(t_{2}, t\right)\right\},
$$

where $W_{j, \tau}$ is given in Eq. (17) and $h\left(t_{j}, t\right)$ is a Gaussian profile centered at $t_{j}$. The canonical dual frames $\tilde{\mathscr{F}}_{\#}^{(a, m)}$ are built as described in Appendix B 3, and are essentially the two-pulse extension of the dual frame shown in Fig. 7(a) therein. 


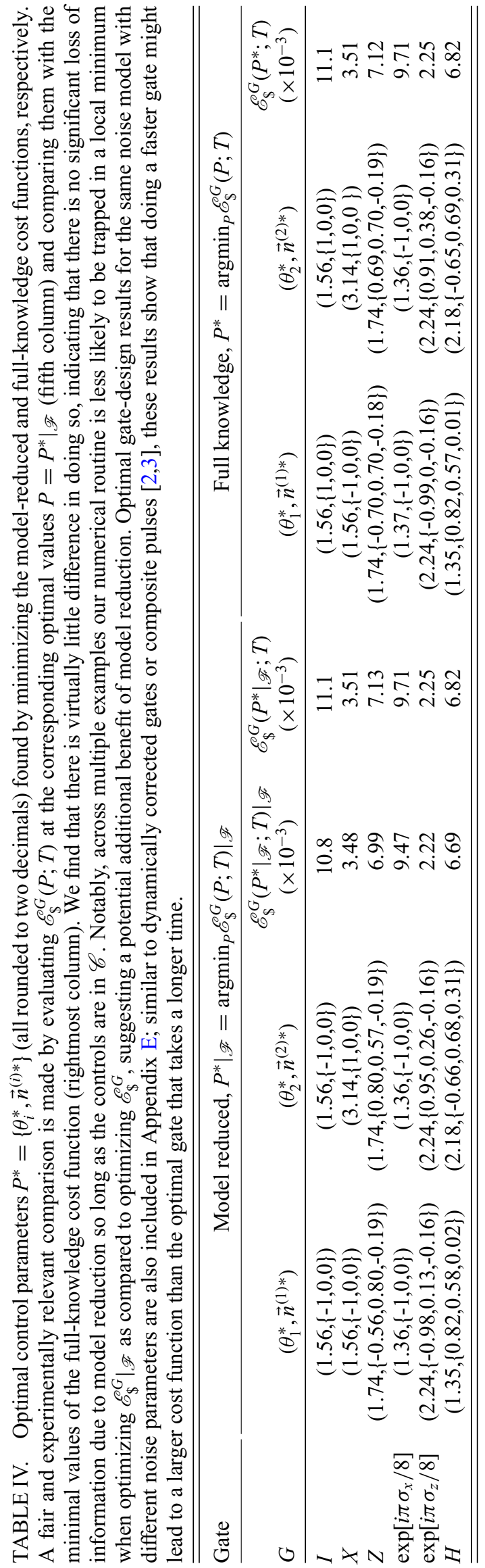

We then perform CA QNS via control sequences obtained from Eq. (16) in the single-qubit case, with pulse directions and angles respectively given by $\vec{n}^{(1)}=\vec{n}^{(2)} \equiv \vec{n}$,

$$
\begin{gathered}
\vec{n} \in\left\{(0,1,0),\left(\frac{1}{\sqrt{2}}, \frac{1}{\sqrt{2}}, 0\right),\left(\frac{1}{\sqrt{2}}, 0, \frac{1}{\sqrt{2}}\right),\left(0, \frac{1}{\sqrt{3}}, \frac{\sqrt{2}}{\sqrt{3}}\right)\right\}, \\
\theta_{1}, \theta_{2} \in\left\{0, \pi, \frac{64 \pi}{35}, \frac{17 \pi}{10}, 2 \pi\right\} .
\end{gathered}
$$

While in principle any "sufficiently large" set of directions and angles works for our purposes, the above set is sufficient to estimate all the necessary CA-spectra components while leading to a well-defined and stable estimation problem. Note that, in principle, one would need to infer the $10 \times 10+2 \times 2=104$ parameters describing $\overline{\mathscr{S}}_{\mathscr{C}}$. However, given a chosen frame, there are necessarily symmetries in the CA spectra. To account for them, we systematically classify these symmetries by a kernel analysis method, as detailed in Appendix D. In our example, this implies that the total number of parameters to infer reduces to $30+2=32$. Our estimation of these parameters, i.e., of $\overline{\mathscr{S}}_{\mathscr{C}}$, is summarized in Fig. 4 (right panels).

\section{Prediction and optimization}

In order to test the ability of our model-reduced representation to predict the dynamics of the system at time $T$, we calculate the distance

$$
\left.\mathscr{E}(P ; T) \equiv \sum_{u, v}\left|e_{u, v}(P ; T)-e_{u, v}(P ; T)\right| \mathscr{F}\right|^{2}
$$

between the model-reduced and full-knowledge predictions for 1000 randomly chosen configurations $P=$ $\left\{\theta_{1}, \theta_{2}, \vec{n}^{(1)}, \vec{n}^{(2)}\right\}$. We find that the average $\mathscr{E}(P ; T)_{\text {avg }}=$ $1.18 \times 10^{-9}$, with a worst case $\mathscr{E}(P ; T)_{\text {worst }}=8.17$ $\times 10^{-8}$. Furthermore, choosing a pair of random pulse directions $\left\{\vec{n}^{(1)}, \vec{n}^{(2)}\right\}$, we evaluate $\mathscr{E}(P ; T)$ for $\left\{\theta_{1}, \theta_{2}\right\}$ in $[0,2 \pi]$. The results are shown in Fig. 4 (bottom left).

Prediction is the precursor to optimization, and we can thus demonstrate the benefits that our model-reduced representation brings to the problem of optimally executing a desired gate $G$ given $\mathscr{C}$. Using standard Nelder-Mead numerical routines, for several representative choices of $G$, we search for the optimal parameters $P^{*}=\left\{\theta_{i}^{*}, \vec{n}^{(i) *}\right\}$ that minimize: (i) the cost function $\left.\mathscr{E}_{\mathbb{S}}^{G}(P ; T)\right|_{\mathscr{F}}$, by using the information $\overline{\mathscr{S}}_{\mathscr{C}}$ inferred in the above characterization stage; or (ii) the cost function $\mathscr{E}_{\mathbb{S}}^{G}(P ; T)$, by assuming full knowledge of the noise model, with access to the fullmodel time-domain equations. Scenario (ii) is a drastic idealization as such knowledge is never available in practice, and no QNS protocol can provide such information unless one assumes arbitrary control capabilities. Nevertheless, it is a useful benchmark, as our objective is to 
show that our model-reduced representation of the dynamics captures all the relevant information, as dictated by $\mathscr{C}$, to a very good approximation.

The results are presented in Table IV. They demonstrate the model reduction capabilities of the formalism, as there is little to no predictive power lost. Note that $\mathscr{E}_{S}^{G}\left(\left.P^{*}\right|_{\mathscr{F}} ; T\right) \sim \mathscr{E}_{S}^{G}\left(P^{*} ; T\right)$, as desired. Finally, for completeness and to highlight the benefits of control, we calculate the value of the cost function $\mathscr{E}_{S} G\left(P_{0}=\left\{\theta_{i}=0\right\} ; t\right)$ for $G=I$ using full information and in the absence of control, i.e., the effect of the natural decoherence of the system. One finds that in the absence of control $\mathscr{E}_{\$} I\left(P_{0} ; T / 2\right)=$ $9.77 \times 10^{-3}$ and $\mathscr{E}_{\$} I\left(P_{0} ; T\right)=9.09 \times 10^{-2}$, which should be contrasted, for example, with the optimal control solution over time $T$, namely $\mathscr{E}_{\$} I\left(P^{*} ; T\right)=11.1 \times 10^{-3}$.

\section{On the universality of QNS-inferred information for control purposes}

Fundamental to the paradigm of $\mathrm{C} \& \mathrm{C}$ of open quantum systems is the assumption that QNS-inferred information is sufficient to implement high-accuracy operations. This, however, is not guaranteed. QNS protocols infer information about the noise by measuring the response of the system to a fixed set of control sequences, say $\mathscr{C}_{0} \subseteq \mathscr{C}$, so, by design, the information they access is only that which these sequences can sense, namely, $\left.\mathscr{S}\right|_{\mathscr{C}_{0}}$. The question is whether this information is universal, that is, whether it can be used to accurately predict the dynamics of the system under a sequence not in $\mathscr{C}_{0}$.

To gain a concrete feeling about this problem, note that standard comb-based QNS protocols [19], but also Slepian-based [20] or spin-locking [15] protocols, sample the leading noise power spectra in the frequency domain at a finite set of points. For control purposes, however, one is interested in overlap integrals of the form given in Table I (panel (iii)); hence, the information provided by the sampling is necessarily incomplete. Therefore, it is necessary to complement it with additional assumptions, by interpolating between the sampled points. The assumptions that are more or less implicitly made in this completion step-e.g., in choosing a particular interpolation method - can be highly arbitrary and user defined, and yet they can decisively influence our ability to predict the dynamics accurately. For instance, given a sampling set, there are in principle infinitely many possible interpolations consistent with it, and it is easy to build a control sequence for which the details of the interpolation are crucial: a simple example demonstrating how the latter can directly impact observable expectation values is given in Fig. 5. That is, $\left.\mathscr{S}\right|_{\mathscr{C}_{0}}$ is not universal in general. Consequently, obtaining rigorous criteria to characterize the control sequences whose effect on the system can be accurately predicted given such information is not only desirable but

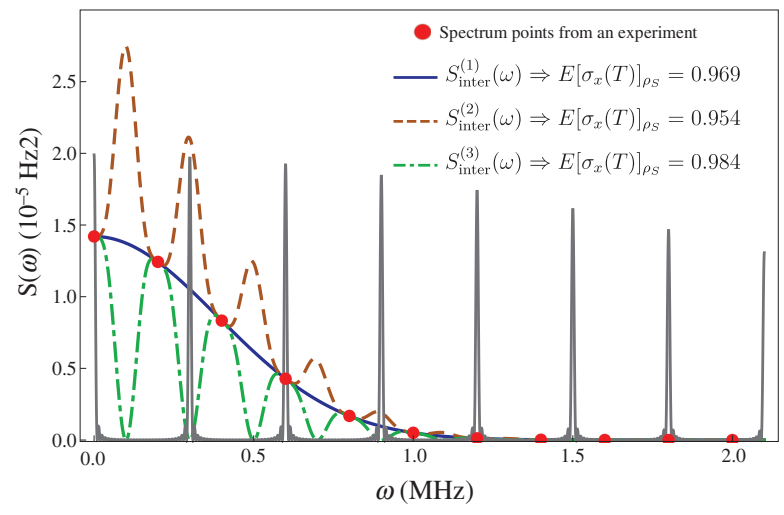

FIG. 5. Illustrative example of the possible issues from incompatibility between a control sequence of interest and QNS-inferred information. Red dots represent sampled points from comb-based SP QNS for a representative Gaussian power spectrum, $S(\omega)=\exp \left[-\omega^{2} /\left(3 \times 10^{11}\right)\right] / 7042$. Shown are also three possible interpolations $S_{\text {inter }}^{(i)}(\omega)$ consistent with them. Namely, (i) $S_{\text {inter }}^{(1)}(\omega)=S(\omega)$ (blue line), (ii) $S_{\text {inter }}^{(2)}(\omega)=S(\omega)$ $\left(1+\sin ^{4}\left[\frac{1}{2} \pi \omega \times 10^{-5}\right]\right) \quad$ (brown dashed line), and (iii) $S_{\text {inter }}^{(3)}(\omega)=S(\omega)\left(1-\sin ^{4}\left[\frac{1}{2} \pi \omega \times 10^{-5}\right]\right)$ (green dash-dot line). Given in the legend are the predicted expectation values of $\sigma_{x}$, obtained by using the initial state $\rho_{S}=\left(\sigma_{0}+\sigma_{x}\right) / 2$ and the expression $E\left[\sigma_{x}(T)\right]_{\rho_{S}}=\exp \left[-\int d \omega\left|F^{(1)}(\omega, T)\right|^{2} S_{\text {inter }}^{(i)}(\omega)\right]$. The predicted value can vary significantly depending on the interpolation being considered if the control sequence being studied is not "of the form" of those used in the QNS protocol. In this case, this means ensuring that $\left|F^{(1)}(\omega, T)\right|^{2}$ (in gray) has peaks out of phase with the interpolation points. An example of such a filter [12] can be generated by $M \gg 1$ repetitions of a $\pi$-pulse sequence with cycle time $T_{c}=(20 \pi) / 3 \mu \mathrm{s}$. Shown here is the filter for $M=10$ repetitions of a Hahn-echo sequence given by $\left[T_{c} / 2-\sigma_{x}-T_{c} / 2-\sigma_{x}\right]$.

also imperative. Of course, this is not a problem exclusive to spectral estimation in either the classical [54-56] or quantum settings, and indeed the task of picking a good interpolation given sampled data is a mainstay in applied mathematics, with various possible criteria available [57].

The frame-based approach we proposed in Sec. III and demonstrated in Sec. IV provides a way around the above problem. On the one hand, by construction, if the finite frame $\mathscr{F}_{\#}$ exists, the FSF condition guarantees that, for an appropriately chosen $\mathscr{C}_{0}$, i.e., a well-designed QNSprotocol, we have $\left.\mathscr{S}\right|_{\mathscr{C}_{0}}=\left.\overline{\mathscr{S}}\right|_{\mathscr{C}}$. Accordingly, the finite frame provides a space in which the (finite) sampling is precisely what is needed to accurately (up to an error scaling with $\varepsilon$ ) predict the behavior of the system under an arbitrary control modulation allowed by $\mathscr{C}$. This naturally obviates the need to complement the sampled noise information, for instance, via interpolation. In the SP frequencydomain example shown in Fig. 5, this would be akin to demanding that all the sequences we are interested in were such that $\sqrt{\int d \omega\left|F(\omega, T)-\sum_{k} F(\omega, T) \delta\left(\omega-k \omega_{0}\right)\right|^{2}} \leq \varepsilon$, 
with $\omega_{0}=2 \pi / T_{c}$. Clearly, such a condition would severely constraint the control sequences we can accurately predict the behavior of. The frame-based approach can achieve the desired effect while overcoming this limitation. Likewise, given an arbitrary control sequence (not necessarily in $\mathscr{C}$ ), one can estimate the prediction error one would incur by using QNS-inferred information $\left.\overline{\mathscr{S}}\right|_{\mathscr{C}}$. Namely, by calculating the distance between the control matrix elements associated with such a sequence and their projection on the frame $\mathscr{F}_{\#}$ associated with the QNS protocol, one essentially obtains an effective $\varepsilon$, and it is then up to the user to determine if such an error is acceptable.

In closing, we note that the question of universality is vital when model-reduced representations of complex systems are introduced (not necessarily related to control). For example, recent work [58] has addressed similar questions, but related to the "objectivity" of classical noise representations of quantum baths. In our setting, if one thinks of the context [58] to be defined by $\mathscr{C}$ then universality of the QNS-inferred information based on $\mathscr{C}_{0}$ may be taken to signify a degree of objectivity of the noise representation for control purposes. Moving forward, it would be interesting to better understand how different model simplifications interact and can lead to more comprehensive model-reduced representations (relative to the combined contexts for example).

\section{CONCLUSION AND OUTLOOK}

We have introduced a framework for constructing a model-reduced representation of open quantum dynamics relative to given control capabilities, which both mathematically formalizes and substantially simplifies the problem of C\&C for general non-Markovian noise environments. While we have exemplified our analysis in two paradigmatic applications - QNS of nonstationary noise and model-reduced $\mathrm{C} \& \mathrm{C}$ of a single qubit - our results also formally justify the success of the machine-learning enhanced approach for noise discrimination proposed in Ref. [59].

Our framework lends itself to several generalizations. On the one hand, a natural and important next step is to develop explicit frame-based protocols applicable to multiqubit $\mathrm{C} \& \mathrm{C}$ tasks in the presence of more general noise models, including multiaxis and non-Gaussian noise. As we mentioned, of special significance in this context will be to understand how, for fixed control capabilities $\mathscr{C}$, a model-reduced description with maximum parsimony may be obtained without sacrificing accuracy [44]. On the other hand, the use of frame-based optimization need not be restricted to the synthesis of unitary target gates; one could imagine leveraging the natural decoherence of the system in the presence of applied control to optimally implement a reachable completely positive trace-preserving map, possibly in connection with ideas from Ref. [60]. Ultimately, we believe that the use of frames will ease the integration of signal processing tools into quantum control and prove instrumental to develop efficient model-reduced approaches to $\mathrm{C} \& \mathrm{C}$ of realistic open quantum systems of growing complexity, as needed by both realistic noisy intermediate-scale quantum era devices and full-fledged fault-tolerant architectures.

\section{ACKNOWLEDGMENTS}

It is a pleasure to thank Howard M. Wiseman, Michael J.W. Hall, and Areeya Chantasri for valuable comments. Work at Griffith was supported by funding from the Australian Government via AUSMURI Grant No. AUSMURI000002. G.P.S. is pleased to acknowledge support from the DECRA fellowship DE170100088. Work at Dartmouth was supported in part by the U.S. Army Research Office through U.S. MURI Grant No. W911NF1810218.

\section{APPENDIX A: TIME-DEPENDENT EXPECTATION VALUES}

As discussed in the main text, in order to capture the system dynamics under the simultaneous effect of the noise and the applied time-dependent, open-loop control, we consider the expectation value of a system-only (invertible) observable $O$, given in the physical frame by $E[O(T)]_{\rho_{S} \otimes \rho_{B}}=\left\langle\operatorname{Tr}\left[U(T)\left(\rho_{S} \otimes \rho_{B}\right) U^{\dagger}(T) O\right]\right\rangle_{c}$. The latter may be rewritten in the form

$$
\begin{aligned}
E[O(T)]_{\rho_{S} \otimes \rho_{B}} & =\left\langle\operatorname{Tr}\left[\widetilde{U}(T)\left(\rho_{S} \otimes \rho_{B}\right) \widetilde{U}^{\dagger}(T) \widetilde{O}(T)\right]\right\rangle_{c} \\
& =\operatorname{Tr}_{S}\left[\left\langle\widetilde{O}(T)^{-1} \widetilde{U}^{\dagger}(T) \widetilde{O}(T) \widetilde{U}(T)\right\rangle \rho_{S} \widetilde{O}(T)\right], \\
& \equiv \operatorname{Tr}\left[V_{O}(T) \rho_{S} \widetilde{O}(T)\right],
\end{aligned}
$$

where $\langle\cdot\rangle_{c}$ represents averaging over realizations of the stochastic process, $\langle\cdot\rangle=\left\langle\operatorname{Tr}_{B}\left[\cdot \rho_{B}\right]\right\rangle_{c}$ is the joint classicalquantum average, and $\widetilde{O}(T)=U_{0}^{\dagger}(T) O U_{0}(T)$. Following a similar line of reasoning as in Ref. [18], we write $V_{O}(T)$ as a time-ordered exponential, $V_{O}(T)=\left\langle\mathcal{T}_{+} e^{-i \int_{-T}^{T} d s H_{O}(s)}\right\rangle$ with

$$
H_{O}(t)= \begin{cases}\bar{H}(T-t), & t \in[0, T], \\ \widetilde{H}(T+t), & t \in[-T, 0],\end{cases}
$$

$\bar{H}(t) \equiv-\widetilde{O}(T)^{-1} \widetilde{H}(t) \widetilde{O}(T)$, and $\widetilde{H}(t)$ being the togglingframe Hamiltonian given in Eq. (3) of the main text. In turn, this allows us to expand $V_{O}(T)$ via a cumulant or Dyson expansion,

$$
\begin{aligned}
\left\langle\mathcal{T}_{+} e^{-i \int_{-T}^{T} H_{O}(t) d t}\right\rangle & =e^{\sum_{k=1}^{\infty}(-i)^{k} \mathcal{C}_{O}^{(k)}(T) / k !} \\
& =1+\sum_{k=1}^{\infty} \frac{\mathcal{D}_{O}^{(k)}(T)}{k !}
\end{aligned}
$$


where $\mathcal{C}_{O}^{(k)}$ is the generalized cumulant defined implicitly as a function of the Dyson-like terms

$$
\begin{aligned}
\frac{\mathcal{D}_{O}^{(k)}(T)}{k !} & =(-i)^{k} \int_{-T}^{T} d_{>} \vec{t}_{[k]}\left\langle H_{O}\left(t_{1}\right) \cdots H_{O}\left(t_{k}\right)\right\rangle \\
& =(-i)^{k} \sum_{\ell=0}^{k} \sum_{\pi \in \Pi_{\ell ; k}} \int_{0}^{T} d_{>} \vec{t}_{[k]} \mathbf{C}_{\ell ; k},
\end{aligned}
$$

with correlators

$$
\mathbf{C}_{\ell ; k} \equiv\left\langle\prod_{j=1}^{\ell} \bar{H}\left(t_{\pi(j)}\right) \prod_{j^{\prime}=\ell+1}^{k} \widetilde{H}\left(t_{\pi\left(j^{\prime}\right)}\right)\right\rangle,
$$

where $d_{>} \vec{t}_{[k]}$ represents time-ordered integration, i.e., such that $t_{1} \geq \cdots \geq t_{k}$. In Eq. (A2), we have performed a change of variables, allowing us to change the integra- tion domain, which leads to the sum over the set $\Pi_{\ell ; k}$, containing the permutations of $\{1, \ldots, k\}$ such that $t_{\pi(1)} \leq$ $\cdots \leq t_{\pi(\ell)}$ and $t_{\pi(\ell+1)} \geq \cdots \geq t_{\pi(k)}$. Expanding each of the correlators, we find that

$$
\begin{aligned}
\mathbf{C}_{\ell ; k} & =(-1)^{\ell} \sum_{\vec{\alpha}, \vec{u}, \vec{v}} f_{\left.\vec{v}\right|_{\ell}}\left\langle\prod_{j=1}^{k} y_{u_{j}, v_{j}}^{\left(\alpha_{j}\right)}\left(t_{\pi(j)}\right) \Lambda_{v_{j}} \otimes B_{u_{j}}^{\left(\alpha_{j}\right)}\left(t_{\pi(j)}\right)\right\rangle \\
& =(-1)^{\ell} \sum_{\vec{\alpha}, \vec{u}, \vec{v}} f_{\left.\vec{v}\right|_{\ell}}^{O} \kappa_{\vec{v}} \hat{\Lambda}_{\vec{v}} \prod_{j=1}^{k} y_{u_{j}, v_{j}}^{\left(\alpha_{j}\right)}\left(t_{\pi(j)}\right)\left\langle\prod_{j=1}^{k} B_{u_{j}}^{\left(\alpha_{j}\right)}\left(t_{\pi(j)}\right)\right\rangle,
\end{aligned}
$$

where $f_{\left.\vec{v}\right|_{\ell}}^{O} \equiv(1 / d) \operatorname{Tr}\left[O^{-1} \Lambda_{v_{1}} \cdots \Lambda_{v_{\ell}} O\left(\Lambda_{v_{1}} \cdots \Lambda_{v_{\ell}}\right)^{-1} \hat{\Lambda}_{\vec{v}}\right]$ and we have assumed for simplicity that the chosen operator basis is such that $\prod_{j=1}^{k} \Lambda_{v_{j}} \equiv \kappa_{\vec{v}} \hat{\Lambda}_{\vec{v}}$ for $\kappa_{\vec{v}} \in \mathbb{C}$ and $\hat{\Lambda}_{\vec{v}}$ invariant under permutations of $\vec{v}$. Finally, this implies that we can write

$$
\begin{aligned}
& \mathcal{D}_{O}^{(k)}(T)=k !(-i)^{k} \sum_{\ell} \sum_{\pi} \sum_{\vec{\alpha}, \vec{u}, \vec{v}}(-1)^{\ell} f_{\vec{v} \mid \ell}^{O} \kappa_{\vec{v}} \hat{\Lambda}_{\vec{v}} \int_{0}^{T} d_{>} \vec{t}_{[k]} \prod_{j=1}^{k} y_{u_{j}, v_{j}}^{\left(\alpha_{j}\right)}\left(t_{\pi(j)}\right)\left\langle\hat{B}_{\vec{u}}^{(\vec{\alpha})}[\pi(\vec{t})]\right\rangle \\
& =k !(-i)^{k} \sum_{\ell}(-1)^{\ell} \sum_{\pi} \sum_{\vec{\alpha}, \vec{u}, \vec{v}} \kappa_{\vec{v}} \hat{\Lambda}_{\vec{v}} \int_{0}^{T} d_{>} \vec{t}_{[k]} \prod_{j=1}^{k} y_{u_{\pi^{-1}(j)}, v_{\pi^{-1}(j)}}^{\left(\alpha_{\pi^{-1}}\right)}\left(t_{j}\right)\left\{f_{\left.\vec{v}\right|_{\ell}}^{O}\left\langle\hat{B}_{\vec{u}}^{(\vec{\alpha})}[\pi(\vec{t})]\right\rangle\right\} \\
& =k !(-i)^{k} \sum_{\ell}(-1)^{\ell} \sum_{\pi} \sum_{\vec{\alpha}, \vec{u}, \vec{v}} \hat{\Lambda}_{\vec{v}} \int_{0}^{T} d_{>} \vec{t}_{[k]} \prod_{j=1}^{k} y_{u_{j}, v_{j}}^{\left(\alpha_{j}\right)}\left(t_{j}\right)\left\{\kappa_{\pi(\vec{v})} f_{\pi(\vec{v}) \mid \ell}^{O}\left\langle\hat{B}_{\pi(\vec{u})}^{(\pi(\vec{\alpha}))}[\pi(\vec{t})]\right\rangle\right\} .
\end{aligned}
$$

From Eq. (A3), obtained by an adequate relabeling of the indices and observing that the sum is over all $\vec{u}, \vec{v}, \vec{\alpha}$, one deduces that, for each configuration of $\alpha_{j}, u_{j}, v_{j}$ in $\{x, y, z\}$, the term $\hat{\Lambda}_{\vec{v}} \prod y_{u_{j} v_{j}}^{\left(\alpha_{j}\right)}\left(t_{j}\right)$ appears modulating a linear combination of bath correlation functions, $\left\langle\hat{B}_{\pi(\vec{u})}^{(\pi(\overrightarrow{)}))}[\pi(\vec{t})]\right\rangle$, i.e., a linear combination $\mathcal{L}_{\vec{\alpha} ; \vec{v}}(\vec{t})$. That is, each of the relevant overlap integrals $\mathcal{I}_{\vec{\alpha} ; \vec{u}, \vec{v}}^{(k)}(T)$ appearing in the sum have the structure claimed in Eq. (6) of the main text. We also highlight that any other perturbative expansion, e.g., a cumulant expansion, can be written in terms of structurally similar overlap integrals. Moreover, any function of the reduced dynamics, e.g., the fidelity, can be expanded in terms of the above integrals and resulting filter function representation. Which function is chosen is then a matter of convenience given the task at hand.

\section{APPENDIX B: ILLUSTRATIVE FRAME EXAMPLES}

\section{Fourier frames and frequency-domain FFs revisited}

The frame formalism encompasses both the Fourier series and the short-time Fourier transform, by respectively relating them to expansions in terms of appropriate discrete Fourier frames [43] or discrete and continuous Gabor frames $[33,61]$. Specifically, the frame of complex exponentials,

$$
\mathscr{F}_{\mathrm{FS}} \equiv\left\{\phi_{n}(t)=e^{-i n 2 \pi t /|\Lambda|}, n \in \mathbb{Z}\right\},
$$

is a discrete, self-dual frame for functions $f \in L^{2}(\Lambda)$, where $\Lambda$ is a closed interval on $\mathbb{R}$ (e.g., $\Lambda=[0,1]$ ) and the inner product $(a, b)=\int_{\Lambda} d t a(t) b(t)^{*} /|\Lambda|$. The resulting frame expansion corresponds to the usual Fourier series on $\Lambda$, namely, $f(t)=\sum_{n}\left(f, \phi_{n}\right) e^{-i n 2 \pi t /|\Lambda|}$ for $t \in \Lambda$.

Likewise, given $f \in L^{2}(\mathbb{R})$, recall that the short-time Fourier transform (STFT, also known as the "windowed" FT or the Weyl-Heisenberg transform) with respect to a window function $g \in L^{2}(\mathbb{R})$ is given by

$$
F_{g}(\omega, \tau) \equiv \int_{-\infty}^{\infty} d t f(t) \phi_{\omega, \tau}^{*}(t), \quad \phi_{\omega, \tau}(t)=g(t-\tau) e^{-i \omega \cdot t}
$$

where $\phi_{\omega, \tau}(t), \omega, \tau \in \mathbb{R}$, are elements of a (continuous) Gabor frame $[61,62]$. That is, a two-dimensional representation of the signal is obtained by taking the FT of 
$f$ as the window function (e.g., a Gaussian or Hanh function centered around zero) is slid along the time axis. Conversely, the inverse STFT is given by $f(t)=$ $\int_{-\infty}^{\infty} d \omega F_{g}(\omega, \tau) \tilde{\phi}_{\omega, \tau}^{*}(t)$, where the functions $\left\{\tilde{\phi}_{\omega, \tau}(t)\right\}$ are dual to $\left\{\phi_{\omega, \tau}(t)\right\}$.

Given the above formalism, one can now see how the standard frequency-domain FF formalism $[34,36]$ constitutes a particular limit of our construction. At an intuitive level, this is possible by considering the FT as an appropriate limit of the Fourier series, in the context of eigenfunction expansions (see, e.g., Sec. 5.7 of Ref. [63]). More formally in our context, the key observation is that any function corresponding to a physically admissible control (including free evolution) is necessarily time limited, i.e., $t_{i} \leq \bar{T}$ for some finite $\bar{T}$ in our overlap integrals. Furthermore, for the SP, one has

$$
\mathcal{I}_{\vec{\alpha} ; \vec{u}, \vec{v}}^{(k)}=\int_{-\infty}^{\infty} d \vec{\tau}_{[k]} \int_{-\infty}^{\infty} d \vec{\omega}_{[k]} F_{\vec{\alpha} ; \vec{u}, \vec{v}}^{(k)}(\vec{\omega}) S_{\vec{\alpha} ; \vec{v}}^{(k)}(\vec{\omega}, \vec{\tau}),
$$

where $S_{\vec{\alpha} ; \vec{v}}^{(k)}(\vec{\omega}, \vec{\tau})=\int_{-\infty}^{\infty} \overrightarrow{d t}_{[k]} \mathcal{L}_{\vec{\alpha}, \vec{v}}(\vec{t}) g(\vec{t}-\vec{\tau}) e^{-i \vec{\omega} \cdot \vec{t}}$ is the FTFT associated with a sliding-window function $g(\vec{t})$ [61]. By noting that, for any $\bar{T}$, there is a $\bar{\tau}$ such that $g(\vec{t}-\vec{\tau})$ and $\prod_{j=1}^{k} y_{u_{j}, v_{j}}^{\left(\alpha_{j}\right)}\left(t_{j}\right)$ have negligible overlap when $|\vec{\tau}|>\bar{\tau}$, one finds that

$$
\mathcal{I}_{\vec{\alpha} ; \vec{u}, \vec{v}}^{(k)} \simeq \int_{|\vec{\tau}| \leq \bar{\tau}} d \vec{\tau}_{[k]} \int_{-\infty}^{\infty} d \vec{\omega}_{[k]} F_{\vec{\alpha} ; \vec{u}, \vec{v}}^{(k)}(\vec{\omega}) S_{\vec{\alpha} ; \vec{v}}^{(k)}(\vec{\omega}, \vec{\tau}) .
$$

In other words, the standard frequency FF formalism is effectively an expansion on the frame given by $\left\{\phi_{\vec{\omega}, \vec{\tau}}\right\}$ for $|\vec{\tau}|<\bar{\tau}$. A similar reasoning can be applied to the CA representation. The two representations are summarized in Table I(iii)-(iv) of the main text.

\section{Digital frames for instantaneous pulses}

Beyond Fourier and Gabor frames, the frame formalism allows for considerable flexibility. Consider the scenario in which the control matrix elements $y_{u, v}(t)$ are piecewise constant in time. As we show in Appendix C, this is relevant, for instance, when one considers $M$ (equidistant) instantaneous pulses over a time $T$, implemented by control profiles $h\left(t_{j}, t\right)=\delta\left[t-\left(t_{j}+\tau / 2\right)\right]$ for $j \in[1, M]$ and $\tau=T / M$. Such control matrix elements are naturally spanned by the sequence $\mathscr{F}_{W}=\left\{W_{j, \tau}\right\}$, where the window function $W_{j, \tau}$ is defined in Eq. (17) in the main text. As it turns out, $\mathscr{F}_{W}$ is not only a frame but also a basis, and for the above scenario, the FSF condition is exactly satisfied $(\varepsilon=0)$. What is more, any digital basis suffices, among which the Walsh functions provide a compelling choice $[46,47]$.

Walsh functions [45] $w_{n}(t)$ are a complete set of orthogonal functions in an interval $[0, T]$ with the inner product $(a, b)=\int_{0}^{T} d t a(t) b(t)^{*} / T$, which form a basis for piecewise constant functions in $[0, T]$ with $2^{N}$ intervals $(N \in$
$\mathbb{N}$ ). They are digital, taking values in $\{-1,1\}$, and can be defined via the rows of the Hadamard matrix $\mathrm{H}_{2^{N}}$. We choose the so-called sequency ordering [45-47] for their labeling for convenience, such that the sequence $\mathscr{F}_{\text {Walsh }_{N}}=\left\{w_{j}(t)\right\}_{j=1}^{2^{N}}$ is a basis for $2^{N}$-interval piecewise constant functions. The first eight Walsh functions are illustrated in Fig. 6.

\section{Custom-built frames for arbitrary pulse profiles}

For general noninstantaneous pulses, corresponding to arbitrary control profiles, one often encounters the situation where the allowed control matrix $\mathscr{Y}$ has a particular structure, e.g., its components are linear combinations of specific functions of the available control profile, and its elements belong to a particular Hilbert space $\mathcal{H}$. Two notable examples, typical of the unitary control scenario resulting from $M$ nonoverlapping pulses in time $T$ we describe in the main text (and in detail in Appendix C), are

$$
\mathscr{Y}^{(a)} \in \mathcal{H}_{\mathscr{Y}^{(a)}}=\operatorname{span}\left\{W_{j, \tau} \cos \left[\theta_{j} \psi_{t}^{(j)}\right], W_{j, \tau} \sin \left[\theta_{j} \psi_{t}^{(j)}\right\}\right.
$$

with $\psi_{t}^{(j)}=\int_{(j-1) \tau}^{t} h\left(t_{j}, s\right) d s$,

$$
\mathscr{Y}^{(m)} \in \mathcal{H}_{\mathscr{Y}(m)}=\operatorname{span}\left\{\theta_{j} h\left(t_{j}, t\right)\right\} .
$$

Consider a generic case where

$$
\mathcal{H}=\operatorname{span}_{\mathbb{C}}\left\{f_{\ell}\left(\left\{\theta_{j}\right\},\left\{b_{j}(t)\right\}\right) \mid \ell \in[1, L], j \in[1, M]\right\}
$$

for some set of functions $\left\{b_{j}(t)\right\}$, e.g., $b_{j}(t)=h\left(t_{j}, t\right)$ as above or where each $b_{j}(t)$ is an element of a convenient (truncated) basis in which control profiles can be expanded. One can build a frame-dual frame pair as follows. Imagine that each $\theta_{j} \in[0,2 \pi]$ takes values among integer multiples of $2 \pi / N_{\text {ctrl }}$ for certain $N_{\text {ctrl }} \in \mathbb{Z}$. Then, the $N_{\#}=N_{\#}\left(L, M, \tilde{N}_{\#}\right)$-element sequence

$$
\mathscr{F}_{\#}=\left\{\phi_{n}\right\} \equiv\left\{f_{\ell}\left(\left\{\eta_{j}\right\},\left\{b_{j}(t)\right\}\right)\right\}, \quad \ell \in[1, L], j \in[1, M],
$$

with $\eta_{i}=2 \pi k_{i} / \tilde{N}_{\#}, k_{i} \in\left[0, \tilde{N}_{\#}\right]$, spans $\mathcal{H}$ when $\tilde{N}_{\#}=N_{\text {ctrl }}$, and is indeed (trivially) a frame. For a different choice of parameter $\tilde{N}_{\#}$, e.g., if $\tilde{N}_{\#}<N_{\text {ctrl }}$, the frame property is lost as $\mathscr{F}_{\#}$ no longer spans $\mathcal{H}$. One can nevertheless proceed to build a dual sequence $\tilde{\mathscr{F}}_{\#}=\left\{\tilde{\phi}_{n}(t)\right\}_{n=1}^{N_{\#}}$ via the MoorePenrose pseudoinverse method (see below), such that, by construction, the orthogonality condition $\left(\phi_{n}, \tilde{\phi}_{n^{\prime}}\right)=\delta_{n, n^{\prime}}$ 


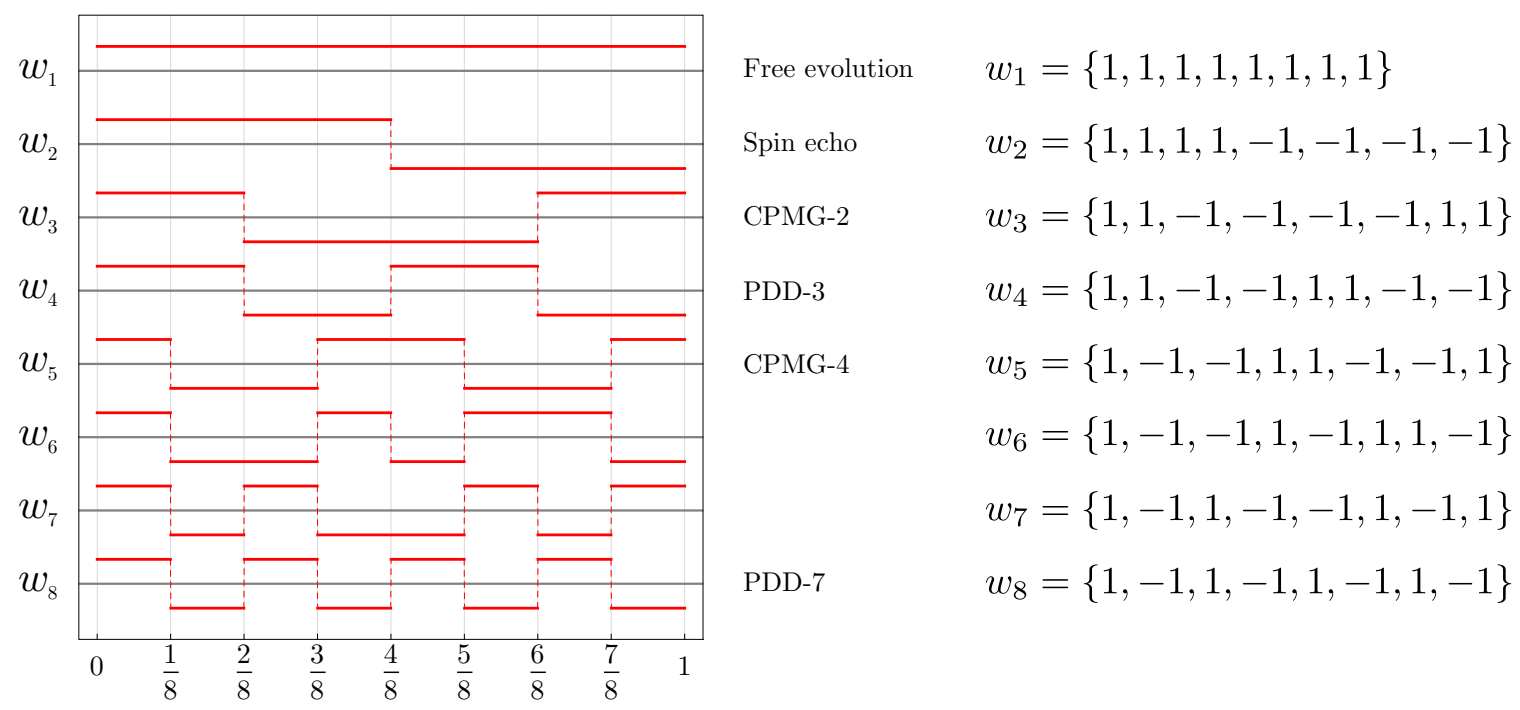

FIG. 6. Left: the first eight Walsh functions in sequency ordering. The gray solid lines represent zero for each function. The names of the traditional $\pi$-pulse sequences $[46,47]$ are written next to the plot. Right: each Walsh function is represented with its values in intervals.

is satisfied. With this, one can write

$$
\tilde{y}(t)=\sum_{n=1}^{N_{\#}}\left(y, \tilde{\phi}_{n}\right) \phi_{n}(t)
$$

and calculate the error bound

$$
\max _{y(t)}\|y(t)-\tilde{y}(t)\|_{2}=\max _{y(t)} \sqrt{\int_{0}^{T}|y(t)-\tilde{y}(t)|^{2} d t} \leq \varepsilon
$$

for the candidate $\mathscr{F}_{\#}$ and $\tilde{\mathscr{F}}_{\#}$, as necessary to verify the FSF condition. That is, one can assess the ability of a candidate frame-dual frame pair to approximate every $y(t) \in$ $\mathcal{H}$ via Eq. (B3), thereby verifying the parsimony of $\mathscr{F}_{\# \text {. }}$

\section{a. Single-qubit additive and multiplicative noise}

For the additive dephasing noise, we study in the main text (see also Appendix C), the five dual frame functions built for $\tilde{N}_{\#}=2$ and $M=1$ are depicted in Fig. 7(a). Here, the associated frame is given by $\mathscr{F}_{\#}^{(a)}=\left\{\phi_{n}^{(a)}(t)\right\}=$ $\left\{1, \cos \left[\pi \psi_{t}^{(1)}\right], \cos \left[2 \pi \psi_{t}^{(1)}\right], \sin \left[\pi \psi_{t}^{(1)}\right], \sin \left[2 \pi \psi_{t}^{(1)}\right]\right\}$, and its dual can be calculated as outlined below. It is worth highlighting that, when the $b_{j}(t)$ are nonoverlapping, as in the $b_{j}(t)=h\left(t_{j}, t\right)$ case considered here, one has $N_{\#}=M L\left(\tilde{N}_{\#}+1\right)$, that is, the size of the frame grows linearly with the number of pulses $M$ (note that the $\phi_{0}^{(a)}=\sin \left[0 \psi_{t}^{(1)}\right]=0$ is trivial and thus excluded from the frame definition, leading to $N_{\#}=5$ ). For any $y(t)$, one can calculate an upper bound $\varepsilon$ to the $L^{2}$ distance $\|y(t)-\tilde{y}(t)\|_{2}$ by evaluating these quantities for any $\theta_{1}=$ $k 2 \pi / 100$ for $k \in[0,100]$. We find that $\left.\varepsilon\right|_{\tilde{N}_{\#}=2}=2.4 \times 10^{-5}$ and $\left.\varepsilon\right|_{\tilde{N}_{\#=4}}=2.8 \times 10^{-8}$. In Fig. 7(b), we plot seven such $y(t)$ (for random $\theta_{1}$ ) and their approximated frame representation functions (B3) for illustration purposes.

For the case of multiplicative noise, note that $\mathcal{H}_{\mathscr{Y}(m)}=$ $\operatorname{span}\left\{h\left(t_{j}, t\right)\right\}$, so we may simply take $\mathscr{F}_{\#}^{(m)} \equiv\left\{h\left(t_{j}, t\right)\right\}$ with $j \in[1, M]$, and trivially satisfy the FSF condition in this way. Also, $\tilde{\mathscr{F}}_{\#}^{(m)}=\mathscr{F}_{\#}^{(m)} / Z_{m}$, where $Z_{m}$ is a normalization factor ensuring that $\int_{0}^{T} d t \phi_{j}^{(m)}(t) \phi_{j^{\prime}}^{(m)}(t) / Z_{m}=\delta_{j, j^{\prime}}$.

\section{b. Building a dual sequence via the Moore-Penrose pseudoinverse}

In practice, given a sequence, one can build a canonical dual one via a standard Moore-Penrose pseudoinverse construction. When the sequence under consideration is a frame, then its dual is also a frame. The starting point is a reference orthonormal basis. We first note that $\mathscr{F}_{\#}=$ $\left\{\phi_{n}(t)\right\}_{n=1, \ldots, N_{\sharp}}$ spans a Hilbert space $\mathcal{H}_{\#}$ with the usual $L^{2}$ inner product. An orthonormal basis for $\mathcal{H}_{\#}$, which we denote by $\mathscr{G}_{\#}=\left\{g_{m}\right\}_{m=1, \ldots, M_{\#}}$ with $M_{\#} \leq N_{\#}$, is then built by applying a Gram-Schmidt process to $\mathscr{F}_{\#}$. In turn, this means that one can write $\phi_{n}=\sum_{m}\left(\phi_{n}, g_{m}\right) g_{m}$, which is represented in matrix language as $\vec{\phi}=\mathbf{T} \vec{g}$, with $\mathbf{T}$ generally a nonsquare matrix. The dual frame can then be built via the Moore-Penrose pseudoinverse. One then has

$$
\begin{aligned}
\phi_{n} & =\sum_{m}\left(\phi_{n}, g_{m}\right) g_{m}=\sum_{n^{\prime}}\left(\phi_{n}, \phi_{n^{\prime}}\right) \tilde{\phi}_{n^{\prime}} \\
& =\sum_{n^{\prime}, m^{\prime}, m, j^{\prime}}\left(\phi_{n}, g_{m}\right)\left(\phi_{n^{\prime}}, g_{m}\right)\left(\tilde{\phi}_{n^{\prime}}, g_{m^{\prime}}\right) g_{m^{\prime}},
\end{aligned}
$$


(a)
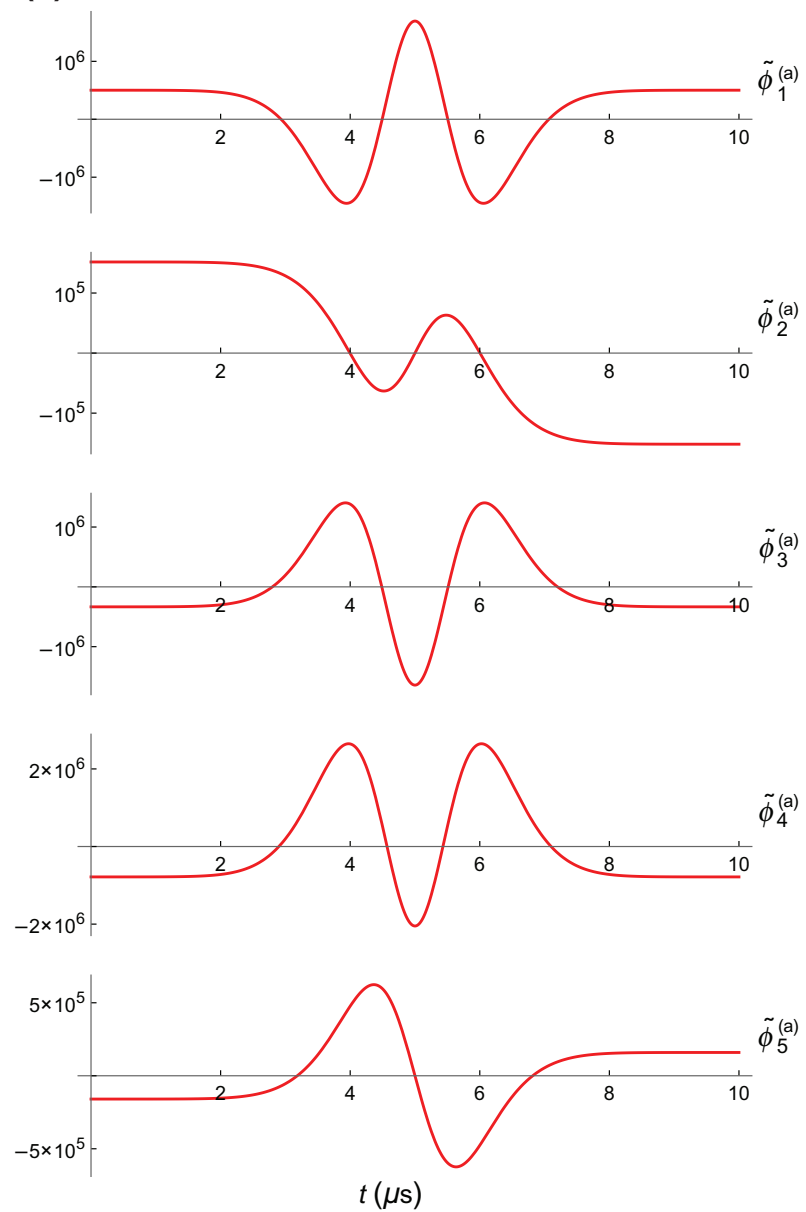

(b)

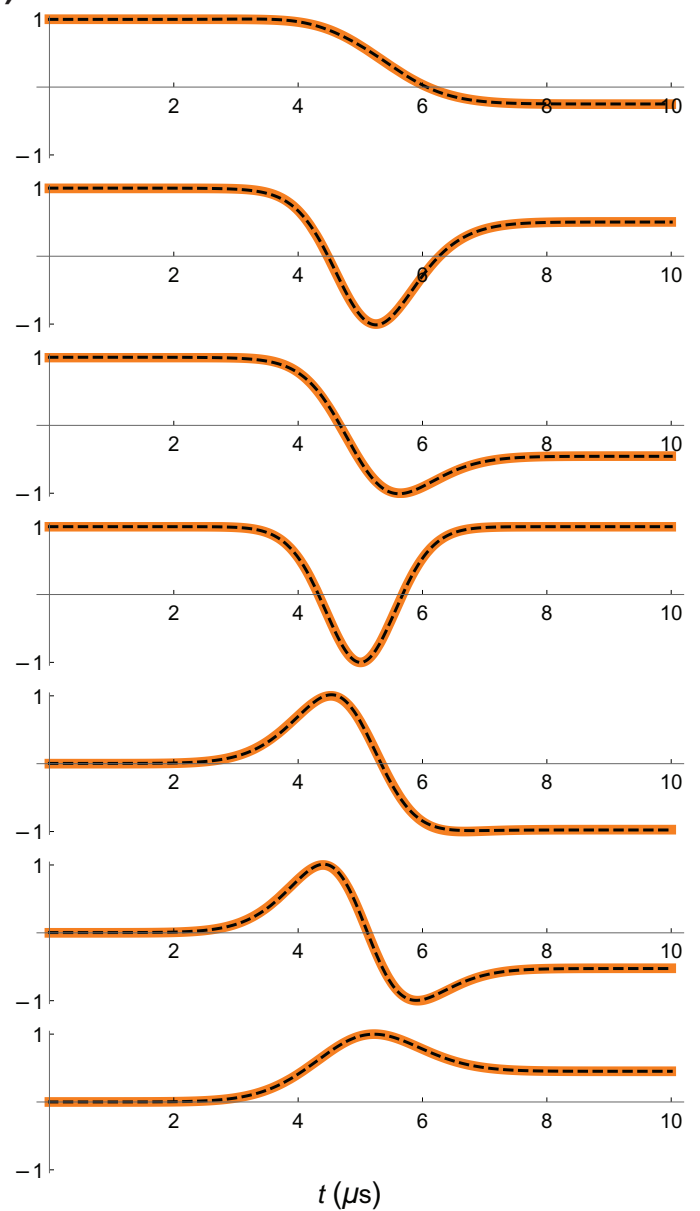

FIG. 7. (a) The five functions in the dual frame $\tilde{\mathscr{F}}^{(a)}$, obtained via the Moore-Penrose pseudoinverse. (b) Seven examples (orange thick) of $\cos \left(\theta_{1} \psi_{t}^{(1)}\right)$ and $\sin \left(\theta_{1} \psi_{t}^{(1)}\right)$ with randomly generated $\theta_{1}$ and their approximated frame representation (black dashed) with $\tilde{N}_{\#}=2$.

where we have used the facts that $\tilde{\phi}_{j}=\sum_{j^{\prime}}\left(\tilde{\phi}_{j}, g_{j^{\prime}}\right) g_{j^{\prime}}$, or, equivalently, $\overrightarrow{\tilde{\phi}}=\tilde{\mathbf{T}} \vec{g}$, and that the $\left\{g_{j^{\prime}}\right\}$ are a basis. The above implies that $\mathbf{T}^{\top} \tilde{\mathbf{T}}=I$, which, noting that $\mathbf{T}^{\top} \mathbf{T}$ is invertible, has the solution $\tilde{\mathbf{T}}=\mathbf{T}\left(\mathbf{T}^{\top} \mathbf{T}\right)^{-1}$. Thus, the elements of $\tilde{\mathscr{F}}_{\text {\# }}$ are given by

$$
\overrightarrow{\tilde{\phi}}=\mathbf{T}\left(\mathbf{T}^{\top} \mathbf{T}\right)^{-1} \vec{g} .
$$

\section{APPENDIX C: SINGLE-QUBIT FRAME-BASED PROTOCOLS}

\section{Single-qubit reduced dynamics and determination of $\overline{\mathscr{S}}_{\mathscr{C}}$}

We are interested in obtaining explicit expressions for expectation values of an invertible observable $O$ at a time $T$. As mentioned in the text, this can be accomplished in a weak-coupling regime via a truncated Dyson expansion given by

$$
\begin{aligned}
E[O(T)]_{\rho_{S} \otimes \rho_{B}} & \approx\left\langle\operatorname{Tr}\left\{\left[I_{S}-\mathcal{D}_{O}^{(2)}(T)\right] \rho_{S} \widetilde{O}(T)\right\}\right\rangle \\
& =\operatorname{Tr}\left[\rho_{S} \widetilde{O}(T)\right]-\left\langle\operatorname{Tr}\left[\mathcal{D}_{O}^{(2)}(T) \rho_{S} \widetilde{O}(T)\right]\right\rangle,
\end{aligned}
$$

where the Dyson term is given by [see Eq. (A3)]

$$
\begin{array}{r}
\mathcal{D}_{O}^{(2)}(T)=2 ! \int_{0}^{T} d_{>} \vec{t}_{[2]}\left\langle\widetilde{H}\left(t_{1}\right) \widetilde{H}\left(t_{2}\right)-\bar{H}\left(t_{2}\right) \widetilde{H}\left(t_{1}\right)\right. \\
\left.-\bar{H}\left(t_{1}\right) \widetilde{H}\left(t_{2}\right)+\bar{H}\left(t_{2}\right) \bar{H}\left(t_{1}\right)\right\rangle_{c}
\end{array}
$$

and $\bar{H}(t) \equiv \sum_{u} y_{u}^{(\alpha)}(t) \sum_{c} f_{c}^{u} \sigma_{c} \otimes B^{(\alpha)}(t) \quad$ with $f_{c}^{u}=\frac{1}{2}$ $\operatorname{Tr}\left[\widetilde{O}^{\dagger}(T) \sigma_{u} \widetilde{O}(T) \sigma_{c}\right]$. Assuming that there are no correlations between additive and multiplicative noise, i.e., $\left\langle B^{(a)}\left(t_{1}\right) B^{(m)}\left(t_{2}\right)\right\rangle=0$ for all $t_{1}, t_{2}$, we have 


$$
\begin{aligned}
\frac{\mathcal{D}_{O}^{(2)}(T)}{2}=\sum_{\alpha ; u, v} \int_{0}^{T} d_{>} \vec{t}_{[2]}[ & y_{u}^{(\alpha)}\left(t_{1}\right) y_{v}^{(\alpha)}\left(t_{2}\right) \sigma_{u} \sigma_{v}\left\langle B^{(\alpha)}\left(t_{1}\right) B^{(\alpha)}\left(t_{2}\right)\right\rangle_{c} \\
& \quad-y_{u}^{(\alpha)}\left(t_{1}\right) y_{v}^{(\alpha)}\left(t_{2}\right)\left(\sum_{c} f_{c}^{u} \sigma_{c}\right) \sigma_{v}\left\langle B^{(\alpha)}\left(t_{1}\right) B^{(\alpha)}\left(t_{2}\right)\right\rangle_{c} \\
& -y_{u}^{(\alpha)}\left(t_{1}\right) y_{v}^{(\alpha)}\left(t_{2}\right)\left(\sum_{c^{\prime}} f_{c^{\prime}}^{v} \sigma_{c^{\prime}}\right) \sigma_{u}\left\langle B^{(\alpha)}\left(t_{2}\right) B^{(\alpha)}\left(t_{1}\right)\right\rangle_{c} \\
& \left.+y_{u}^{(\alpha)}\left(t_{1}\right) y_{v}^{(\alpha)}\left(t_{2}\right) \sum_{c, c^{\prime}} f_{c}^{u} f_{c^{\prime}}^{v} \sigma_{c^{\prime}} \sigma_{c}\left\langle B^{(\alpha)}\left(t_{2}\right) B^{(\alpha)}\left(t_{1}\right)\right\rangle_{c}\right],
\end{aligned}
$$

which, in the frame language, reads

$$
\begin{aligned}
\frac{\mathcal{D}_{O}^{(2)}(T)}{2}= & \frac{\sigma_{0}}{4} \sum_{\alpha ; u ; n, n^{\prime} ; \mu= \pm} \bar{S}_{\alpha}^{(\mu)}\left(n, n^{\prime}\right) F_{\alpha ; u}^{(1,-)}(n, T) F_{\alpha ; u}^{(1,-\mu)}\left(n^{\prime}, T\right) \\
& +\sum_{\alpha ; u \neq v ; n, n^{\prime} ; \mu= \pm} \frac{\sigma_{u} \sigma_{v}}{4} \bar{S}_{\alpha}^{(\mu)}\left(n, n^{\prime}\right) F_{\alpha ; u}^{(1,-)}(n, T) F_{\alpha ; u}^{(1, \mu)}\left(n^{\prime}, T\right)
\end{aligned}
$$

The last equation follows after the change of variables $c \leftrightarrow u, c^{\prime} \leftrightarrow v$ and using $F_{\alpha, u}^{(1, \pm)}(n, T)$ to denote the frame $\mathscr{F}^{(\alpha)}$ representation of $Y_{\alpha, u}^{( \pm)}(t) \equiv y_{u}^{(\alpha)}(t) \pm \sum_{c} f_{c}{ }^{u} y_{c}^{(\alpha)}(t)$, with the definition for $\bar{S}_{\alpha}^{( \pm)}\left(n, n^{\prime}\right)$ used in the main text. Therefore, the second term in Eq. (C1) contains the effect of the noise that we are interested in. In the following, we isolate the quantities $\operatorname{Tr}\left[\mathcal{D}_{O}^{(2)}(T) \sigma_{l}\right], l \in\{x, y, z\}$, for a given control sequence, initial system state, and measured observable, so that we can build our CA-QNS protocol by cycling over a sufficiently large set of controls and observables. First we note that

$$
\begin{aligned}
& E[O(T)]_{\left(I_{S}+\sigma_{k}\right) / 2 \otimes \rho_{B}}+E[O(T)]_{\left(I_{S}-\sigma_{k}\right) / 2 \otimes \rho_{B}}=-\left\langle\operatorname{Tr}\left[\mathcal{D}_{O}^{(2)}(T) \widetilde{O}(T)\right]\right\rangle, \\
& E[O(T)]_{\left(I_{S}+\sigma_{k}\right) / 2 \otimes \rho_{B}}-E[O(T)]_{\left(I_{S}-\sigma_{k}\right) / 2 \otimes \rho_{B}}=\operatorname{Tr}\left[\sigma_{k} \widetilde{O}(T)\right]-\left\langle\operatorname{Tr}\left[\mathcal{D}_{O}^{(2)}(T) \sigma_{k} \widetilde{O}(T)\right]\right\rangle,
\end{aligned}
$$

which implies that from the (measured) expectation values we can infer the value of $\left\langle\operatorname{Tr}\left[\mathcal{D}_{O}^{(2)}(T) \sigma_{r} \widetilde{O}(T)\right]\right\rangle_{c}$ for $r \in\{0, x, y, z\}$. Then, we note that, for a choice of control and observable, the operator $\widetilde{O}(T)=\sum_{k} \frac{1}{2} \operatorname{Tr}\left[\widetilde{O}(T) \sigma_{k}\right] \sigma_{k} \equiv$ $\sum_{k} o_{k} \sigma_{k}$ is known and fixed, which allows us to write the system of equations

$$
\left\{\operatorname{Tr}\left[\mathcal{D}_{O}^{(2)}(T) \sigma_{r} \widetilde{O}(T)\right]=\sum_{l=0, x, y, z} \sum_{k=x, y, z} \operatorname{Tr}\left[\mathcal{D}_{O}^{(2)}(T) \sigma_{l}\right] o_{k} \frac{\operatorname{Tr}\left[\sigma_{l} \sigma_{r} \sigma_{k}\right]}{2}\right\}_{r \in\{0, x, y, z\}},
$$

from which the $\left\{\operatorname{Tr}\left[\mathcal{D}_{O}^{(2)}(T) \sigma_{l}\right]\right\}$ can be inferred, as desired.

We then combine these values - for a fixed $U_{0}(T)$ - to construct $\operatorname{Tr}\left[\mathcal{D}_{O}^{(2)}(T) \sigma_{r} \widetilde{O}(T)\right]$ for $\widetilde{O}(T)=\sigma_{\gamma}$ and all $r, \gamma$, which simplifies the $\widetilde{O}(T)$-dependent expression for $F_{\alpha, u}^{(1, \pm)}(n, T)$. A direct calculation for $r=0$ shows that

$$
\begin{aligned}
\left\langle\operatorname{Tr}\left[\mathcal{D}_{O}^{(2)}(T) \sigma_{0} \sigma_{\gamma}\right]\right\rangle_{c} & =\sum_{\alpha ; n, n^{\prime}} \sum_{u \neq v \neq \gamma} \sum_{\mu= \pm} i \epsilon_{u v \gamma} \bar{S}_{\alpha}^{(\mu)}\left(n, n^{\prime}\right) F_{\alpha ; u}^{(1,-)}(n, T) F_{\alpha ; v}^{(1, \mu)}\left(n^{\prime}, T\right) \\
& =\sum_{\alpha ; n, n^{\prime}} \sum_{u \neq v \neq \gamma} \sum_{\mu= \pm} i \epsilon_{u v \gamma}\left(1+\mu g_{v}^{\gamma}\right)\left(1-g_{u}^{\gamma}\right) F_{\alpha ; u}^{(1)}(n, T) F_{\alpha ; v}^{(1)}\left(n^{\prime}, T\right) \bar{S}_{\alpha}^{(\mu)}\left(n, n^{\prime}\right) \\
& =4 i \sum_{\alpha ; n, n^{\prime}} \sum_{u \neq v \neq \gamma} \epsilon_{u v \gamma} F_{\alpha ; u}^{(1)}(n, T) F_{\alpha ; v}^{(1)}\left(n^{\prime}, T\right) \bar{S}_{\alpha}^{(-)}\left(n, n^{\prime}\right) \\
& =4 i \sum_{\alpha ; u<v \neq \gamma} \epsilon_{u v \gamma} \overline{\mathcal{I}}_{\alpha ; u, v}^{(2,-)}(T),
\end{aligned}
$$


where $\epsilon_{u v \gamma}$ is the Levi-Civita symbol, $g_{u}^{v}=\frac{1}{2} \operatorname{Tr}\left[\sigma_{u} \sigma_{v} \sigma_{u} \sigma_{v}\right]$, and

$$
\overline{\mathcal{I}}_{\alpha ; u, v}^{(2,-)}(T) \equiv \sum_{n, n^{\prime}}\left[\bar{S}_{\alpha}^{(-)}\left(n, n^{\prime}\right)-\bar{S}_{\alpha}^{(-)}\left(n^{\prime}, n\right)\right] F_{\alpha ; u}^{(1)}(n, T) F_{\alpha ; v}^{(1)}\left(n^{\prime}, T\right)
$$

Similarly, for $r \neq 0$, we find that

$$
\begin{aligned}
\left\langle\operatorname{Tr}\left[\mathcal{D}_{O}^{(2)}(T) \sigma_{r} \sigma_{\gamma}\right]\right\rangle_{c} & \\
= & \sum_{\alpha ; n, n^{\prime}} \sum_{u} \sum_{\mu= \pm} \delta_{u, \gamma}\left(1-g_{u}^{\gamma}\right)\left(1-\mu g_{u}^{\gamma}\right) F_{\alpha ; u}^{(1)}(n, T) F_{\alpha ; u}^{(1)}\left(n^{\prime}, T\right) \bar{S}_{\alpha}^{(\mu)}\left(n, n^{\prime}\right) \\
& +\sum_{\alpha ; n, n^{\prime}} \sum_{u \neq v} \sum_{\mu= \pm}\left(\delta_{r, v} \delta_{\gamma, u}-\delta_{r, u} \delta_{\gamma, v}\right)\left(1-g_{u}^{\gamma}\right)\left(1+\mu g_{v}^{\gamma}\right) F_{\alpha ; u}^{(1)}(n, T) F_{\alpha ; v}^{(1)}\left(n^{\prime}, T\right) \bar{S}_{\alpha}^{(\mu)}\left(n, n^{\prime}\right) \\
= & 4 \sum_{\alpha ; n, n^{\prime}} \sum_{u \neq \gamma}\left[\delta_{r, \gamma} F_{\alpha ; u}^{(1)}(n, T) F_{\alpha ; u}^{(1)}\left(n^{\prime}, T\right) \bar{S}_{\alpha}^{(+)}\left(n, n^{\prime}\right)-\delta_{r, u} F_{\alpha ; u}^{(1)}(n, T) F_{\alpha ; \gamma}^{(1)}\left(n^{\prime}, T\right) \bar{S}_{\alpha}^{(+)}\left(n, n^{\prime}\right)\right] \\
= & 4 \sum_{\alpha ; u \neq \gamma}\left[\delta_{r, \gamma} \mathcal{I}_{\alpha ; u, u}^{(2,+)}(T)-\delta_{r, u} \mathcal{I}_{\alpha ; u, \gamma}^{(2,+)}(T)\right],
\end{aligned}
$$

where the index structure forbids the contribution from $\bar{S}_{\alpha}^{(-)}\left(n, n^{\prime}\right)$, and we have defined

$$
\mathcal{I}_{\alpha ; u, v}^{(2,+)}(T) \equiv \sum_{n, n^{\prime}} \bar{S}_{\alpha}^{(+)}\left(n, n^{\prime}\right) F_{\alpha ; u}^{(1)}(n, T) F_{\alpha ; v}^{(1)}\left(n^{\prime}, T\right)
$$

Therefore, from the possible $r, \gamma$ configurations, and

$$
\left\langle\operatorname{Tr}\left[\mathcal{D}_{O}^{(2)}(T) \sigma_{r} \sigma_{\gamma}\right]\right\rangle_{c}= \begin{cases}4 \sum_{\alpha ; u<v \neq \gamma} i \epsilon_{u v \gamma} \overline{\mathcal{I}}_{\alpha ; u, v}^{(2,-)}(T), & r=0, \\ 4 \sum_{\alpha ; u \neq \gamma} \mathcal{I}_{\alpha ; u, u}^{(2,+)}, & r=\gamma, \\ -4 \sum_{\alpha ; u \neq \gamma} \delta_{u, r} \mathcal{I}_{\alpha ; u, \gamma}^{(2,+)}, & r \neq \gamma, r \neq 0,\end{cases}
$$

we conclude that only the integrals $\mathcal{I}_{\alpha ; u, v}^{(2,+)}$ for all $u, v$ and $\overline{\mathcal{I}}_{\alpha ; u, v}^{(2,-)}$ for all $u \neq v$ influence the reduced qubit dynamics. In turn, this implies that only the quantities $\left.\overline{\mathscr{S}}\right|_{\mathscr{C}}=$ $\left\{\bar{S}_{\alpha}^{(+)}\left(n, n^{\prime}\right), \bar{S}_{\alpha}^{(-)}\left(n, n^{\prime}\right)-\bar{S}_{\alpha}^{(-)}\left(n^{\prime}, n\right)\right\}$ are relevant to the dynamics given $\mathscr{C}$. The objective of QNS is to precisely extract all the spectra in $\left.\overline{\mathscr{S}}\right|_{\mathscr{C}}$.

\section{Control-adapted QNS protocol with instantaneous control}

In the case of instantaneous control, note that each switching function is exactly expanded by an appropriate digital basis, as mentioned in Sec. IV A 2. We thus use Walsh functions (see Appendix B 2) as our frame. To perform CA QNS for such a frame, it is enough to use rotations around the $y$ axis. In this situation, the toggling-frame
Hamiltonian specializes to

$$
\widetilde{H}(t)=y_{z, z}(t) \sigma_{z} \otimes B(t)+y_{z, x}(t) \sigma_{x} \otimes B(t),
$$

where the control matrix elements are such that in the $j$ th time interval they are given by

$$
\begin{aligned}
y_{z, z}(t) & =\cos \left(\tilde{\theta}_{j}\right) \\
& =\frac{1}{2} \operatorname{Tr}\left[e^{i \tilde{\theta}_{j} \sigma_{y} / 2} \sigma_{z} e^{-i \tilde{\theta}_{j} \sigma_{y} / 2} \sigma_{z}\right], \quad(j-1) \tau \leq t<\tau, \\
y_{z, x}(t) & =-\sin \left(\tilde{\theta}_{j}\right) \\
& =\frac{1}{2} \operatorname{Tr}\left[e^{i \tilde{\theta}_{j} \sigma_{y} / 2} \sigma_{z} e^{-i \tilde{\theta}_{j} \sigma_{y} / 2} \sigma_{x}\right], \quad(j-1) \tau \leq t<\tau,
\end{aligned}
$$

with the relation between the $\tilde{\theta}_{j}$ and $\theta_{j}$ set by the equations $0=\tilde{\theta}_{1}, \quad \theta_{k}=\tilde{\theta}_{k+1}-\tilde{\theta}_{k}, \quad 1 \leq k \leq M-1, \quad \theta_{M}=-\tilde{\theta}_{M}$. 
The above considerations suggest the following. First, using only $\tilde{\theta}=\pi$ pulses, one can ensure that $y_{z}(t)$ and $y_{x}(t)$ take values in $\{-1,1\}$, and equal (up to a sign) to any desired Walsh function $w_{n}(t)$ for $t \in[0, T]$ and $n \in[1, M]$. This implies, for example, that

$$
F_{z}^{(1)}(n, T)=\int_{0}^{T} d t w_{m}(t) w_{n}(t)=T \delta_{m n}
$$

and, thus,

$$
\begin{aligned}
\mathcal{I}_{z, z}^{(+)}(T) & \equiv \mathcal{I}_{a ; z, z}^{(2,+)}(T) \\
& =T^{2} \sum_{n, n^{\prime}} \bar{S}^{(+)}\left(n, n^{\prime}\right) \delta_{n m} \delta_{n^{\prime} m} \\
& =T^{2} \bar{S}^{(+)}(m, m) .
\end{aligned}
$$

That is, we can directly sample diagonal elements $\bar{S}^{(+)}(m, m)$.

Second, using $\tilde{\theta} \in\{\pi, \pi / 2\}$, we ensure that $y_{z}(t)$ and $y_{x}(t)$ take values in $\{-1,0,1\}$, with the constraint $\left|y_{z}(t)\right|^{2}+$ $\left|y_{x}(t)\right|^{2}=1$. In particular, one can choose angles such that $y_{z}(t)=w_{m}(t)+w_{m^{\prime}}(t)$, while $y_{x}(t)=w_{m}(t)-w_{m^{\prime}}(t)$ and, thus,

$$
\begin{aligned}
F_{z}^{(1)}(n, T) & =\int_{0}^{T} d t \frac{1}{2}\left[w_{m}(t)+w_{m^{\prime}}(t)\right] w_{n}(t) \\
& =\frac{T}{2}\left(\delta_{n m}+\delta_{n m^{\prime}}\right), \\
F_{x}^{(1)}(n, T) & =\int_{0}^{T} d t \frac{1}{2}\left[w_{m}(t)-w_{m^{\prime}}(t)\right] w_{n}(t) \\
& =\frac{T}{2}\left(\delta_{n m}-\delta_{n m^{\prime}}\right) .
\end{aligned}
$$

When applied to our dynamical equations, the above implies that

$$
\begin{aligned}
\mathcal{I}_{z, z}^{(+)}(T)= & \frac{T^{2}}{4}\left[\bar{S}^{(+)}(m, m)+\bar{S}^{(+)}\left(m, m^{\prime}\right)\right. \\
& \left.+\bar{S}^{(+)}\left(m^{\prime}, m\right)+\bar{S}^{(+)}\left(m^{\prime}, m^{\prime}\right)\right], \\
\mathcal{I}_{z, x}^{(+)}(T)= & \frac{T^{2}}{4}\left[\bar{S}^{(+)}(m, m)-\bar{S}^{(+)}\left(m, m^{\prime}\right)\right. \\
& \left.+\bar{S}^{(+)}\left(m^{\prime}, m\right)-\bar{S}^{(+)}\left(m^{\prime}, m^{\prime}\right)\right], \\
\overline{\mathcal{I}}_{z, x}^{(-)}(T)= & -\frac{T^{2}}{2}\left[\bar{S}^{(-)}\left(m, m^{\prime}\right)-\bar{S}^{(-)}\left(m^{\prime}, m\right)\right],
\end{aligned}
$$

and we can thus infer the elements $\bar{S}^{(+)}\left(n, n^{\prime}\right)$ and $\bar{S}^{(-)}\left(n, n^{\prime}\right)-\bar{S}^{(-)}\left(n^{\prime}, n\right)$, as desired.

Given access to the corresponding $\left.\overline{\mathscr{S}}\right|_{\mathscr{C}}$ and noting that the SP and the CA pictures are related via $S^{( \pm)}\left(n, n^{\prime}\right)=$
$\bar{S}^{( \pm)}\left(n, n^{\prime}\right) \pm \bar{S}^{( \pm)}\left(n^{\prime}, n\right)$, one can then obtain Walsh reconstructions $\hat{C}_{ \pm}^{(a)}\left(t_{1}, t_{2}\right)$ of $C_{ \pm}^{(a)}\left(t_{1}, t_{2}\right)$ given by

$$
\hat{C}_{ \pm}^{(a)}\left(t_{1}, t_{2}\right)=\sum_{n, n^{\prime}=1}^{N_{\#}}\left[\bar{S}^{( \pm)}\left(n, n^{\prime}\right) \pm \bar{S}^{( \pm)}\left(n^{\prime}, n\right)\right] w_{n}\left(t_{1}\right) w_{n^{\prime}}\left(t_{2}\right) .
$$

The reconstruction resolution will depend on the free parameters in the above protocol, namely, the total time $T$ and the minimum switching time $\tau$, which upper bounds the value of $N_{\#}$. In general, a smaller $\tau$ leads to higher resolution.

\section{APPENDIX D: SYMMETRY ANALYSIS FOR CONTROL-ADAPTED SPECTRA}

Given the frame of choice as plotted in Fig. 8, there are symmetries in the CA spectra. We systematically classify any symmetries present in $\bar{S}_{a}^{+}\left(n, n^{\prime}\right)$ by a kernel analysis method (which also works for more general noise models) as follows.

(1) The relevant set of parameters, $\bar{S}_{a}^{+}\left(n, n^{\prime}\right)$, are not linearly independent. To probe this, we decompose each of them by dividing the integration region $0 \leq$ $t_{2} \leq t_{1} \leq T$ into three distinct subregions, namely $I_{1} \equiv\left\{0 \leq t_{2} \leq t_{1} \leq T / 2\right\}, \quad I_{2} \equiv\left\{T / 2 \leq t_{2} \leq t_{1} \leq T\right\}$, and $I_{3} \equiv\left\{0 \leq t_{2} \leq T / 2, T / 2 \leq t_{1} \leq T\right\}$, and, thus, letting $\left.\bar{S}_{a}^{+}\left(n, n^{\prime}\right)\right|_{i}$ be the component of $\bar{S}_{a}^{+}\left(n, n^{\prime}\right)$ in the $I_{i}$ integration subregion, such that

$$
\bar{S}_{a}^{+}\left(n, n^{\prime}\right)=\left.\bar{S}_{a}^{+}\left(n, n^{\prime}\right)\right|_{1}+\left.\bar{S}_{a}^{+}\left(n, n^{\prime}\right)\right|_{2}+\left.\bar{S}_{a}^{+}\left(n, n^{\prime}\right)\right|_{3} .
$$

The key point is that this division allows the systematic study of the symmetries in the frame elements within each as well as between different integration subregions, e,g., the stationary assumption implies that $\left.\bar{S}_{a}^{+}\left(n, n^{\prime}\right)\right|_{1}=\left.\bar{S}_{a}^{+}\left(m, m^{\prime}\right)\right|_{2}$ when $\phi_{n}^{(a)}\left(t_{1}-T / 2\right)=\phi_{m}^{(a)}\left(t_{1}\right)$ and $\phi_{n^{\prime}}^{(a)}\left(t_{2}-T / 2\right)=\phi_{m^{\prime}}^{(a)}$ $\left(t_{2}\right)$. Moreover, parity symmetries lead to further reduction in the free parameters, e.g., $\left.\bar{S}_{a}^{+}\left(n, n^{\prime}\right)\right|_{1}=$ $-\left.\bar{S}_{a}^{+}\left(n^{\prime}, n\right)\right|_{1}$ when $\phi_{n}^{(a)}\left(t_{1}\right)$ is an odd function in $0 \leq t_{1} \leq T / 2$ (antisymmetric about $t_{1}=T / 4$ ) and $\phi_{n^{\prime}}^{(a)}\left(t_{2}\right)$ is an even function in $0 \leq t_{2} \leq T / 2$ (symmetric about $\left.t_{2}=T / 4\right)$. We then associate a vector $s\left(n, n^{\prime}\right)$ to each $\bar{S}_{a}^{+}\left(n, n^{\prime}\right)$ such that the lth entry, $s\left(n, n^{\prime}\right)_{l}$, is nonzero if $\bar{S}_{a}^{+}\left(n, n^{\prime}\right)$ has a nonzero projection on the $l$ th element of the set of independent elements $\left\{\left.\bar{S}_{a}^{+}\left(n, n^{\prime}\right)\right|_{i}\right\}$ (after considering all the symmetries above), and zero otherwise. Furthermore, we use all elements of the vectors $s\left(n, n^{\prime}\right)$ to construct the matrix

$$
S_{K} \equiv\left(\begin{array}{ccc}
s(1,1)_{1} & s(1,2)_{1} & \cdots \\
s(1,1)_{2} & s(1,2)_{2} & \cdots \\
\vdots & \vdots &
\end{array}\right)
$$


TABLE V. Symmetry equation list. Numbers 1 to 45 list all the combinations of $\left(j_{1}, k_{1}\right)$ and $\left(j_{2}, k_{2}\right)$ such that $\bar{S}_{a}^{+}\left(j_{1}, k_{1}\right) \pm$ $\bar{S}_{a}^{+}\left(j_{2}, k_{2}\right)=0$. Numbers 46 to 70 list all the $\left(j_{3}, k_{3}\right)$ such that $\bar{S}_{a}^{+}\left(j_{3}, k_{3}\right)=0$.

\begin{tabular}{|c|c|c|c|}
\hline 1 & $(1,1)-(6,6)$ & 36 & $(6,2)+(7,1)$ \\
\hline 2 & $(1,2)+(7,6)$ & 37 & $(6,3)-(8,1)$ \\
\hline 3 & $(1,3)-(8,6)$ & 38 & $(6,4)-(9,1)$ \\
\hline 4 & $(1,4)-(9,6)$ & 39 & $(6,5)+(10,1)$ \\
\hline 5 & $(1,5)+(10,6)$ & 40 & $(7,3)+(8,2)$ \\
\hline 6 & $(6,7)+(7,6)$ & 41 & $(7,4)+(9,2)$ \\
\hline 7 & $(6,8)-(8,6)$ & 42 & $(7,5)-(10,2)$ \\
\hline 8 & $(6,9)-(9,6)$ & 43 & $(8,4)-(9,3)$ \\
\hline 9 & $(6,10)+(10,6)$ & 44 & $(8,5)+(10,3)$ \\
\hline 10 & $(2,1)-(7,6)$ & 45 & $(9,5)+(10,4)$ \\
\hline 11 & $(2,2)-(7,7)$ & 46 & $(1,6)$ \\
\hline 12 & $(2,3)+(8,7)$ & 47 & $(1,7)$ \\
\hline 13 & $(2,4)+(9,7)$ & 48 & $(1,8)$ \\
\hline 14 & $(2,5)-(10,7)$ & 49 & $(1,9)$ \\
\hline 15 & $(7,8)+(8,7)$ & 50 & $(1,10)$ \\
\hline 16 & $(7,9)+(9,7)$ & 51 & $(2,6)$ \\
\hline 17 & $(7,10)-(10,7)$ & 52 & $(2,7)$ \\
\hline 18 & $(3,1)-(8,6)$ & 53 & $(2,8)$ \\
\hline 19 & $(3,2)-(8,7)$ & 54 & $(2,9)$ \\
\hline 20 & $(3,3)-(8,8)$ & 55 & $(2,10)$ \\
\hline 21 & $(3,4)-(9,8)$ & 56 & $(3,6)$ \\
\hline 22 & $(3,5)+(10,8)$ & 57 & $(3,7)$ \\
\hline 23 & $(8,9)-(9,8)$ & 58 & $(3,8)$ \\
\hline 24 & $(8,10)+(10,8)$ & 59 & $(3,9)$ \\
\hline 25 & $(4,1)-(9,6)$ & 60 & $(3,10)$ \\
\hline 26 & $(4,2)-(9,7)$ & 61 & $(4,6)$ \\
\hline 27 & $(4,3)-(9,8)$ & 62 & $(4,7)$ \\
\hline 28 & $(4,4)-(9,9)$ & 63 & $(4,8)$ \\
\hline 29 & $(4,5)+(10,9)$ & 64 & $(4,9)$ \\
\hline 30 & $(9,10)+(10,9)$ & 65 & $(4,10)$ \\
\hline 31 & $(5,1)-(10,6)$ & 66 & $(5,6)$ \\
\hline 32 & $(5,2)-(10,7)$ & 67 & $(5,7)$ \\
\hline 33 & $(5,3)-(10,8)$ & 68 & $(5,8)$ \\
\hline 34 & $(5,4)-(10,9)$ & 69 & $(5,9)$ \\
\hline 35 & $(5,5)-(10,10)$ & 70 & $(5,10)$ \\
\hline
\end{tabular}

Since the kernel of $S_{K}$ represents the vanishing linear combinations of its columns, calculating it provides exactly the linear dependencies among $\left\{\bar{S}_{a}^{+}\left(n, n^{\prime}\right)\right\}$ we seek. For our choice of frame, we find 70 such symmetries (see Table V), and thus the number of free parameters - which describe the additive noise - is now reduced from 100 to 30 .

(2) Recalling that, for multiplicative noise, the $j$ th frame element is supported on the $j$ th interval, one then has $\bar{S}_{m}^{+}\left(j, j^{\prime}\right)=0$ for $j<j^{\prime}$ and $\bar{S}_{m}^{+}(j, j)=$ $\bar{S}_{m}^{+}\left(j^{\prime}, j^{\prime}\right)$.

(3) Finally, we point out that the additive and multiplicative noise components can be separately inferred by first fixing $\left\{\theta_{i}\right\}$ and cycling over a sufficiently large set of directions $\vec{n}$, and then repeating these steps for several choices of $\left\{\theta_{i}\right\}$.
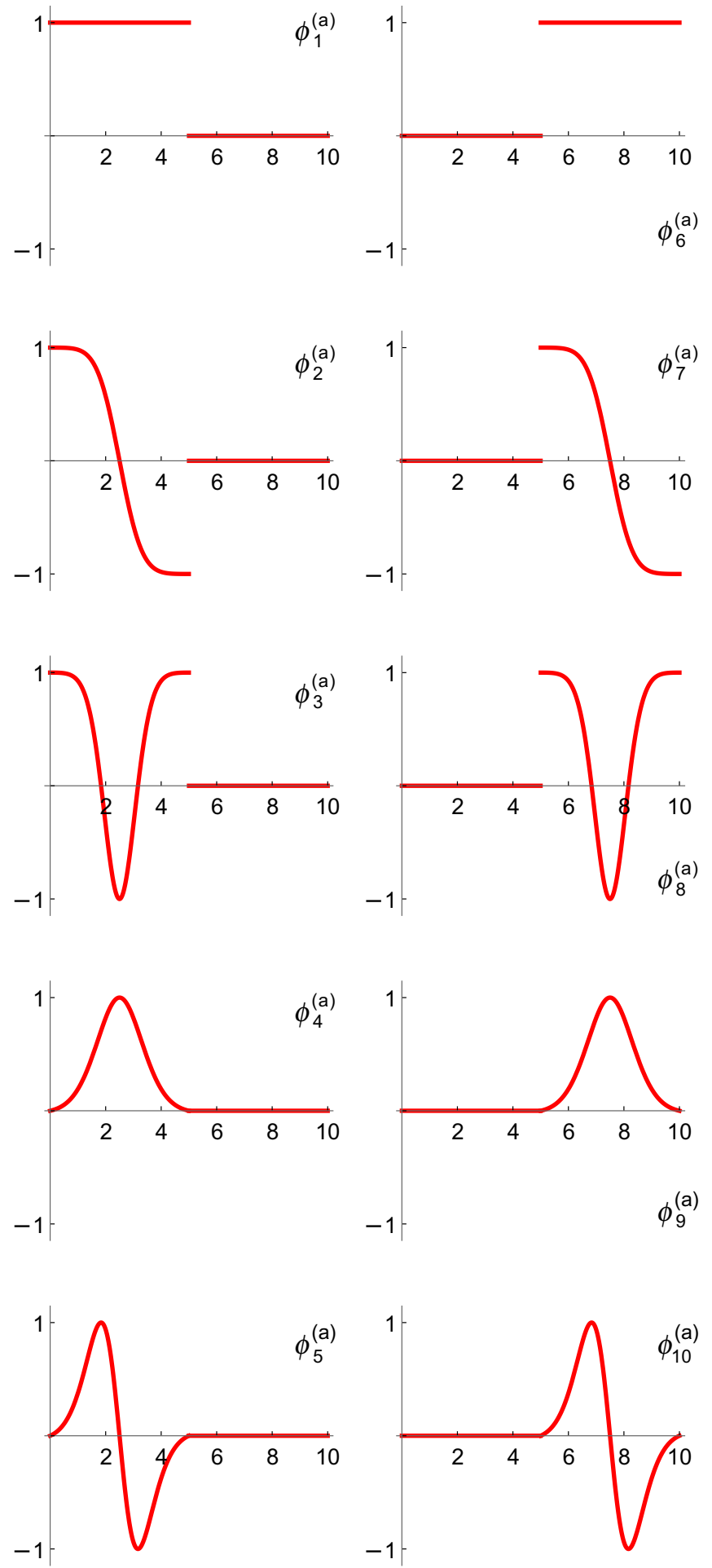

FIG. 8. Frame elements in $\mathscr{F}_{\#}^{(a)}$ for $M=2$ and $\tilde{N}_{\#}=2$. Horizontal axes are time, $t(\mu s)$.

\section{APPENDIX E: FASTER GATES NEED NOT BE MORE ACCURATE}

Here we showcase another noise example where twointerval control can, somewhat surprisingly, lead to better 


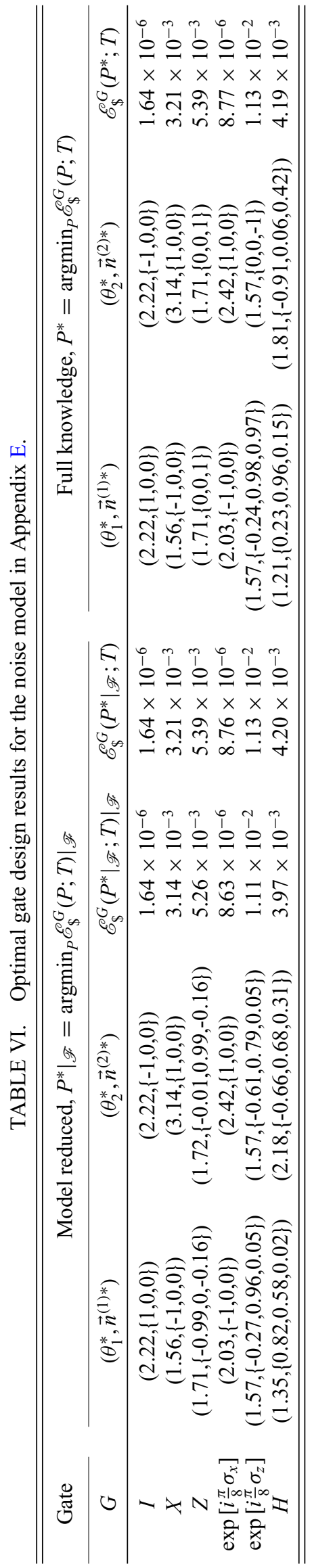

performance in some gate design tasks than single-interval control. This noise model is the same as that of Sec. IV C 1, except that the parameter values are changed as $b_{0}^{(a)} / \hbar=$ $2000 \mathrm{kHz}, c_{0}^{(a)}=0.08 \mathrm{~ms}^{2}, b_{1}^{(a)} / \hbar=5 \times 10^{4} \mathrm{kHz}, c_{1}^{(a)}=$ $0.64 \mathrm{~s}^{2}, \omega_{1}^{(a)}=400 \mathrm{kHz}, b_{0}^{(m)} / \hbar=0.1 \mathrm{mHz}, c_{0}^{(m)} / \hbar=$ $6 \sqrt{2 \pi} \mathrm{Hz}$, and $\omega_{0}^{(m)}=60 \mathrm{~Hz}$. The optimal gate design results are summarized in Table VI. In comparison, the shortest implementation of the $\pi / 8$ gate around $X$ allowed by $\mathscr{C}$ yields the larger error $\mathscr{E}_{S}^{G}(\{\pi / 4\} ; T / 2)=7.75 \times$ $10^{-3}$. As in dynamically corrected gates [2] and composite pulses [3], multiple segments of evolution may be crucial to enable error cancelation, despite the gate taking longer.

[1] L. Viola, E. Knill, and S. Lloyd, Dynamical Decoupling of Open Quantum Systems, Phys. Rev. Lett. 82, 2417 (1999); L. Viola and E. Knill, Robust dynamical decoupling of quantum systems with bounded controls, ibid. 90, 037901 (2003).

[2] K. Khodjasteh and L. Viola, Dynamically Error-Corrected Gates for Universal Quantum Computation, Phys. Rev. Lett. 102, 080501 (2009); K. Khodjasteh, D. A. Lidar, and L. Viola, Arbitrarily accurate dynamical control in open quantum systems, ibid. 104, 090501 (2010); K. Khodjasteh, H. Bluhm, and L. Viola, Automated synthesis of dynamically corrected quantum gates, Phys. Rev. A 86, 042329 (2012).

[3] C. Kabytayev, T. J. Green, K. Khodjasteh, M. J. Biercuk, L. Viola, and K. R. Brown, Robustness of composite pulses to time-dependent control noise, Phys. Rev. A 90, 012316 (2014).

[4] G. A. Paz-Silva, S.-W. Lee, T. J. Green, and L. Viola, Dynamical decoupling sequences for multi-qubit dephasing suppression and long-time quantum memory, New J. Phys. 18, 073020 (2016).

[5] Y. Wang, M. Um, J. Zhang, S. An, M. Lyu, J.-N. Zhang, L.-M. Duan, D. Yum, and K. Kim, Single-qubit quantum memory exceeding ten-minute coherence time, Nat. Photonics 11, 646 (2017).

[6] G. Gordon, G. Kurizki, and D. A. Lidar, Optimal Dynamical Decoherence Control of a Qubit, Phys. Rev. Lett. 101, 010403 (2008).

[7] C. Brif, R. Chakrabarti, and H. Rabitz, Control of quantum phenomena: Past, present and future, New J. Phys. 12, 075008 (2010).

[8] C. P. Koch, Controlling open quantum systems: Tools, achievements, and limitations, J. Phys.: Cond. Mat. 28, 213001 (2016).

[9] M. M. Müller, S. Gherardini, T. Calarco, S. Montangero, and F. Caruso, Information-theoretic limits for quantum optimal control solutions: Error scaling of noisy channels, arXiv:2006.16113 (2020).

[10] C. L. Degen, F. Reinhard, and P. Cappellaro, Quantum sensing, Rev. Mod. Phys. 89, 035002 (2017).

[11] F. Beaudoin, L. M. Norris, and L. Viola, Ramsey interferometry in correlated quantum noise environments, Phys. Rev. A 98, 020102 (2018). 
[12] G. A. Álvarez and D. Suter, Measuring the Spectrum of Colored Noise by Dynamical Decoupling, Phys. Rev. Lett. 107, 230501 (2011).

[13] T. Yuge, S. Sasaki, and Y. Hirayama, Measurement of the Noise Spectrum using a Multiple-Pulse Sequence, Phys. Rev Lett. 107, 170504 (2011).

[14] F. Yan, J. Bylander, S. Gustavsson, F. Yoshihara, K. Harrabi, D. G. Cory, T. P. Orlando, Y. Nakamura, J.-S. Tsai, and W. D. Oliver, Spectroscopy of low-frequency noise and its temperature dependence in a superconducting qubit, Phys. Rev. B 85, 174521 (2012).

[15] F. Yan, S. Gustavsson, J. Bylander, X. Jin, F. Yoshihara, D. G. Cory, Y. Nakamura, T. P. Orlando, and W. D. Oliver, Rotating-frame relaxation as a noise spectrum analyser of a superconducting qubit undergoing driven evolution, Nat. Commun. 4, 2337 (2013).

[16] O. Dial, M. D. Shulman, S. P. Harvey, H. Bluhm, V. Umansky, and A. Yacoby, Charge Noise Spectroscopy Using Coherent Exchange Oscillations in a Singlet-Triplet Qubit, Phys. Rev. Lett. 110, 146804 (2013).

[17] L. M. Norris, G. A. Paz-Silva, and L. Viola, Qubit Noise Spectroscopy for Non-Gaussian Dephasing Environments, Phys. Rev. Lett. 116, 150503 (2016).

[18] G. A. Paz-Silva, L. M. Norris, and L. Viola, Multiqubit spectroscopy of Gaussian quantum noise, Phys. Rev. A 95, 022121 (2017).

[19] P. Szańkowski, G. Ramon, J. Krzywda, D. Kwiatkowski, and $\mathrm{L}$. Cywiński, Environmental noise spectroscopy with qubits subjected to dynamical decoupling, J. Phys. C 29, 333001 (2017); J. Krzywda, P. Szańkowski, and Ł. Cywiński, The dynamical-decoupling-based spatiotemporal noise spectroscopy, New J. Phys. 21, 043034, (2019); G. Ramon, Trispectrum reconstruction of non-Gaussian noise, Phys. Rev. B 100, 161302 (2019).

[20] V. M. Frey, S. Mavadia, L. M. Norris, W. de Ferranti, D. Lucarelli, L. Viola, and M. J. Biercuk, Application of optimal band-limited control protocols to quantum noise sensing, Nat. Commun. 8, 2189 (2017);; L. M. Norris, D. Lucarelli, V. M. Frey, S. Mavadia, M. J. Biercuk, and L. Viola, Optimally band-limited spectroscopy of control noise using a qubit sensor, Phys. Rev. A 98, 032315 (2018);; V. M. Frey, L. M. Norris, L. Viola, and M. J. Biercuk, Simultaneous Spectral Estimation of Dephasing and Amplitude Noise on a Qubit Sensor by Optimally BandLimited Control, Phys. Rev. Appl. 14, 024021 (2020).

[21] K. Willick, D. K. Park, and J. Baugh, Efficient continuouswave noise spectroscopy beyond weak coupling, Phys. Rev. A 98, 013414 (2018).

[22] C. Ferrie, C. Granade, G. Paz-Silva, and H. M. Wiseman, Bayesian quantum noise spectroscopy, New J. Phys. 20, 123005 (2018).

[23] K. W. Chan, W. Huang, C. H. Yang, J. C. C. Hwang, B. Hensen, T. Tanttu, F. E. Hudson, K. M. Itoh, A. Laucht, A. Morello, and A. S. Dzurak, Assessment of a Silicon Quantum Dot Spin Qubit Environment via Noise Spectroscopy, Phys. Rev. Appl. 10, 044017 (2018).

[24] G. A. Paz-Silva, L. M. Norris, F. Beaudoin, and L. Viola, Extending comb-based spectral estimation to multiaxis quantum noise, Phys. Rev. A 100, 042334 (2019).
[25] Y.-Q. Chen, K.-L. Ma, Y.-C. Zheng, J. Allcock, S. Zhang, and C.-Y. Hsieh, Non-Markovian Noise Characterization with the Transfer Tensor Method, Phys. Rev. Appl. 13, 034045 (2020).

[26] U. von Lüpke, F. Beaudoin, L. M. Norris, Y. Sung, R. Winik, J. Y. Qiu, M. Kjaergaard, D. Kim, J. Yoder, S. Gustavsson, L. Viola, and W. D. Oliver, Two-Qubit Spectroscopy of Spatio-Temporally Correlated Quantum Noise in Superconducting Qubits, Phys. Rev. X Quantum 1, 010305 (2020).

[27] A. Chakrabarti, I. Chakraborty, and R. Bhattacharyya, Dynamic decoupling in the presence of 1D random walk, J. Stat. Mech. 2016, 053210 (2016).

[28] T. Proctor, M. Revelle, E. Nielsen, K. Rudinger, D. Lobser, P. Maunz, R. Blume-Kohout, and K. Young, Detecting and tracking drift in quantum information processors, Nat. Commun. 11, 5396 (2020).

[29] R. J. Duffin and A. C. Schaeffer, A class of nonharmonic Fourier series, T. Am. Math. Soc. 72, 341 (1952).

[30] J. Kovačević and A. Chebira, An Introduction to Frames, Found. Trends ${ }^{\circledR}$ Signal Proc. 2, 1 (2008).

[31] P. G. Casazza, G. Kutyniok, and F. Philipp, in Finite Frames: Theory and Applications, edited by P. G. Casazza and G. Kutyniok (Birkhauser Boston, Boston, 2013), p. 1.

[32] S. T. Ali, J.-P. Antoine, and J.-P. Gazeau, Continuous frames in Hilbert space, Ann. Phys. 222, 1 (1993).

[33] M. Fornasier and H. Rauhut, Continuous frames, function spaces, and the discretization problem, J. Fourier Anal. Appl. 11, 245 (2005).

[34] A. G. Kofman and G. Kurizki, Unified Theory of Dynamically Suppressed Qubit Decoherence in Thermal Baths, Phys. Rev. Lett. 93, 130406 (2004).

[35] M. Biercuk, A. Doherty, and H. Uys, Dynamical decoupling sequence construction as a filter-design problem, J. Phys. B 44, 154002 (2011).

[36] G. A. Paz-Silva and L. Viola, General Transfer-Function Approach to Noise Filtering in Open-Loop Quantum Control, Phys. Rev. Lett. 113, 250501 (2014).

[37] N. Didier, E. A. Sete, J. Combes, and M. P. da Silva, Ac Flux Sweet Spots in Parametrically Modulated Superconducting Qubits, Phys. Rev. Appl. 12, 054015 (2019).

[38] G. A. Paz-Silva, M. J. W. Hall, and H. M. Wiseman, Dynamics of initially correlated open quantum systems: Theory and applications, Phys. Rev. A 100, 042120 (2019).

[39] R. Kubo, Generalized cumulant expansion method, J. Phys. Soc. Jpn. 17, 1100 (1962).

[40] N. J. Munch, Noise reduction in tight Weyl-Heisenberg frames, IEEE Trans. Inf. Theory 38, 608 (1992).

[41] J. M. Renes, R. Blume-Kohout, A. J. Scott, and C. M. Caves, Symmetric informationally complete quantum measurements, J. Math. Phys. 45, 2171 (2004); A. Bisio, G. Chiribella, G. M. D’Ariano, S. Facchini, and P. Perinotti, Optimal quantum tomography, IEEE J. Sel. Top. Quantum Elec. 15, 1646 (2009).

[42] C. Ferrie and J. Emerson, Frame representations of quantum mechanics and the necessity of negativity in quasiprobability representations, J. Phys. A 41, 352001 (2008). 
[43] J. Ortega-Cerdà and K. Seip, Fourier frames, Ann. Math. 155, 789 (2002).

[44] G. Obinata and B. D. Anderson, Model Reduction for Control System Design (Springer-Verlag, London, 2001).

[45] J. L. Walsh, A closed set of normal orthogonal functions, Am. J. Math. 45, 5 (1923).

[46] D. Hayes, K. Khodjasteh, L. Viola, and M. J. Biercuk, Reducing sequencing complexity in dynamical quantum error suppression by Walsh modulation, Phys. Rev. A 84, 062323 (2011); H. Qi, J. Dowling, and L. Viola, Optimal digital dynamical decoupling for general decoherence via Walsh modulation, Quantum Inf. Proc. 16, 272 (2017).

[47] A. Cooper, E. Magesan, H. N. Yum, and P. Cappellaro, Time-resolved magnetic sensing with electronic spins in diamond, Nat. Commun. 5, 3141 (2014).

[48] Throughout the manuscript we calculate the relative error between an approximated/estimated quantity $\hat{A}$ and the actual one $A$, by $\mathcal{E}_{\text {relative }}=\|\hat{A}-A\| /\|A\|$.

[49] D. Stanek, B. Fauseweh, C. Stihl, S. Pasini, and G. S. Uhrig, Anomalous behavior of control pulses in presence of noise with singular autocorrelation, J. Magn. Res. 245, 133 (2014).

[50] K. Khodjasteh, T. Erdélyi, and L. Viola, Limits on preserving quantum coherence using multipulse control, Phys. Rev. A 83, 020305 (2011).

[51] A. Napolitano, Cyclostationary Processes and Time Series (Academic Press, London, San Diego, Cambridge, Oxford, 2019).

[52] C. Quintana et al., Observation of Classical-Quantum Crossover of 1/f Flux Noise and its Paramagnetic Temperature Dependence, Phys. Rev. Lett. 118, 057702 (2017).
[53] E. Barnes, F. A. Calderon-Vargas, W. Dong, B. Li, J. Zeng, and F. Zhuang, Dynamically corrected gates from geometric space curves, arXiv:2103.16015 (2021).

[54] A. Høst-Madsen and C. Caspersen, Spectral estimation for random sampling using interpolation, Signal Process. 46, 297 (1995).

[55] C. I. Byrnes, T. T. Georgiou, and A. Lindquist, A new approach to spectral estimation: A tunable high-resolution spectral estimator, IEEE Trans. Signal Process. 48, 3189 (2000).

[56] A. A. Minda, C.-I. Barbinta, and G.-R. Gillich, A review of interpolation methods used for frequency estimation, Romanian J. Acoust. Vib. 17, 21 (2020).

[57] C. Caruso and F. Quarta, Interpolation methods comparison, Comput. Math. Appl. 35, 109 (1998).

[58] P. Szańkowski and Ł. Cywiński, Noise representations of open system dynamics, Sci. Rep. 10, 22189 (2020); P. Szańkowski, Measuring trajectories of environmental noise, arXiv:2106.08434 (2021).

[59] A. Youssry, G. A. Paz-Silva, and C. Ferrie, Characterization and control of open quantum systems beyond quantum noise spectroscopy, npj Quantum Inf. 6, 95 (2020).

[60] F. Ticozzi and L. Viola, Quantum and classical resources for unitary design of open-system evolutions, Quantum Sci. Tech. 2, 034001 (2017).

[61] K. Gröchenig, Foundations of Time-Frequency Analysis (Birkhäuser, Boston, 2001).

[62] in: Practical Time-Frequency Analysis, Wavelet Analysis and Its Applications, edited by R. Carmona, W.-L. Hwang, and B. Torrésani (Academic Press, 1998), Vol. 9, p. 101.

[63] F. W. Byron and R. W. Fuller, Mathematics of Classical and Quantum Physics (Dover, New York, 1992). 